\title{
Shock Radiation Tests for Saturn and Uranus Entry Probes
}

\author{
Brett A. Cruden, David W. Bogdanoff \\ ERC, Inc.at NASA Ames, Moffett Field, CA 94035
}

This paper describes a test series in the Electric Arc Shock Tube at NASA Ames Research Center with the objective of quantifying shock-layer radiative heating magnitudes for future probe entries into Saturn and Uranus atmospheres. Normal shock waves are measured in Hydrogen-Helium mixtures (89:11\% by volume) at freestream pressures between 13-66 Pa (0.1-0.5 Torr) and velocities from 20-30 km/s. No shock layer radiation is detected within measurement limits below $25 \mathrm{~km} / \mathrm{s}$, a finding consistent with predictions for Uranus entries. Between $25-30 \mathrm{~km} / \mathrm{s}$, radiance is quantified from the Vacuum Ultraviolet through Near Infrared, with focus on the Lyman- $\alpha$ and Balmer series lines of Hydrogen. Shock profiles are analyzed for electron number density and electronic state distribution. The shocks do not equilibrate over several $\mathrm{cm}$, and in many cases the state distributions are non-Boltzmann. Radiation data are compared to simulations of Decadal Survey entries for Saturn and shown to be as much as $8 \times$ lower than predicted with the Boltzmann radiation model. Radiance is observed in front of the shock layer, the characteristics of which match the expected diffusion length.

\section{Nomenclature}

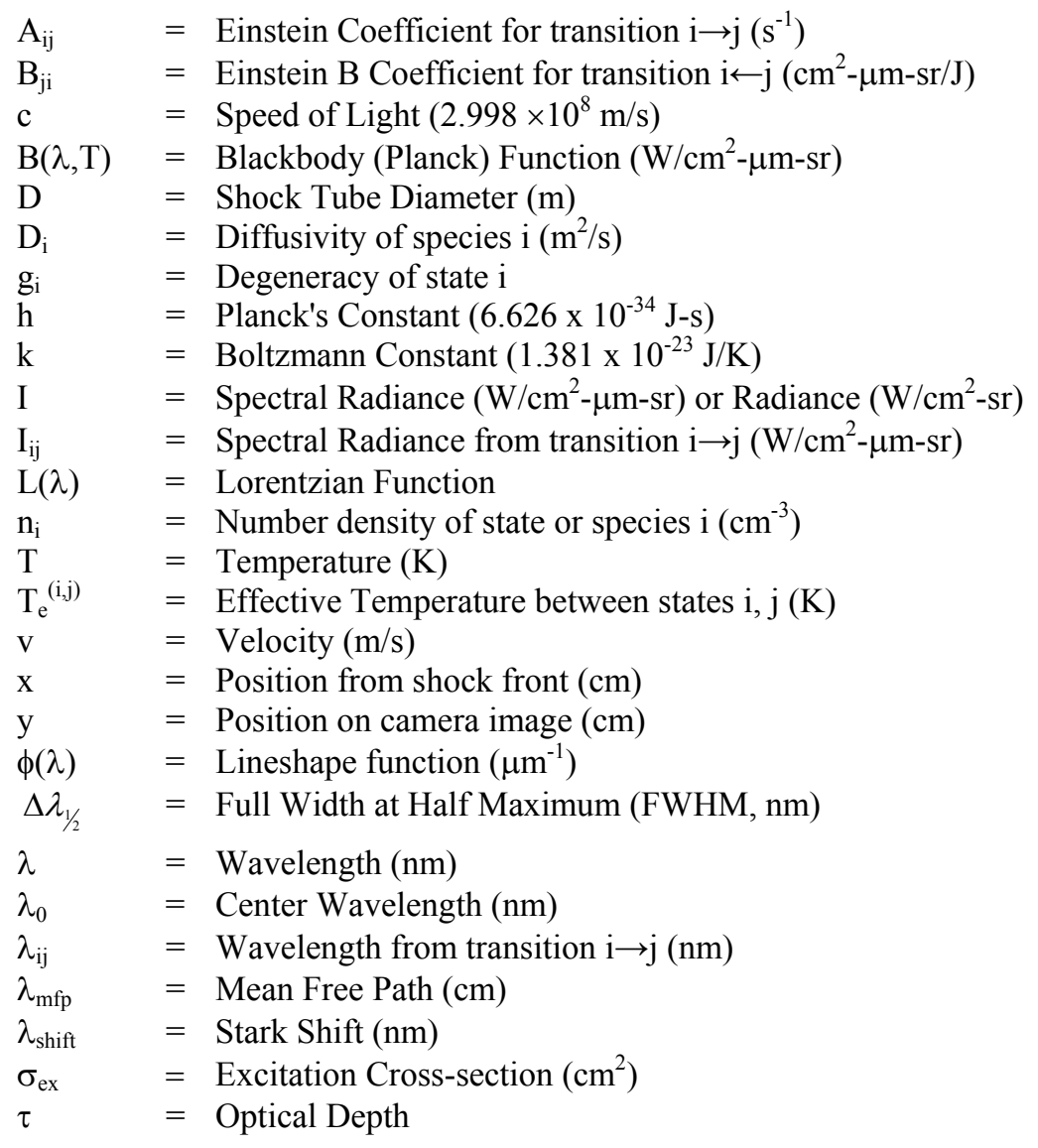




\section{Introduction}

$\mathrm{T}$ HE 2013-2022 Decadal Survey for planetary exploration has identified probe missions to Uranus and Saturn as high priorities [1]. Recently, using the state-of-the-art simulation tools at NASA Ames Research Center, uncertainties in predictive models for these entries were evaluated through Monte Carlo analysis [2]. For both cases, the uncertainty in convective heating was shown to be relatively low. For Uranus, the entry velocities and shocklayer temperatures are low enough that radiative heating is negligible. For Saturn entries however, depending on trajectory, the radiative heating could make up a significant fraction (up to $20 \%$ ) of peak heating. Furthermore, the uncertainty in radiative heating from the CFD model alone (i.e. not including radiative modeling uncertainty) was substantial, with the range of Monte Carlo solutions extending from $0.5-4 \times$ the nominal value. The dominant error source in this study was identified as the $\mathrm{H}$ atom recombination (equivalently $\mathrm{H}_{2}$ dissociation) rate, which impacted the non-equilibrium $\mathrm{H}_{2}$ radiance.

A shock tube test in the Electric Arc Shock Tube (EAST) facility was therefore constructed to examine this radiative heating uncertainty, with the results reported in this work. $\mathrm{H}_{2}$-He mixtures have been studied in the Electric Arc Shock Tube previously in the 1970s for Galileo (Jupiter) entry [3], in 1998-99 in support of the Giant Planet Facility, and in 2004-05 for Neptune Aerocapture [4]. In these latter tests, electron densities within the shock were found to be much larger than expected from equilibrium considerations. The conjecture was that the radiation from the arc driver could heat the shock to a temperature well above that predicted by equilibrium shock wave theory [5]. Consequently, much of the data previously collected in $\mathrm{H}_{2}$-He mixtures in the EAST facility is of questionable value. Approaches to mitigate the driver heating phenomenon were successfully employed and are described in this paper. The paper also describes the radiative measurements at flight relevant conditions, analysis of spectral data, and recommendations for improving radiative heating models for Giant Planet Entries.

\section{Approach}

\section{A. Experimental}

The EAST facility has been described in detail in previous work [6], and is summarized here. The facility consists of a driver, a driven tube (with optional buffer section), and a dump tank (Figure 1). The EAST electric arc driver is conical with a $10.16 \mathrm{~cm}$ exit diameter and $1292 \mathrm{~cm}^{3}$ volume. The cross-wire grounding configuration discussed in the work of Dannenberg [7] was used for conditions reported here. The energy for the arc discharge is supplied by a $1.2 \mathrm{MJ}, 40 \mathrm{kV}$ capacitor bank with a total capacitance of $1530 \mu \mathrm{F}$. The buffer is a $3 \mathrm{~m}$ section of $10.16 \mathrm{~cm}$ tube that is located between, and isolated from, the driven and driver gas. Helium gas was chosen for both the driver and the buffer so as to avoid a resonance excitation of the Hydrogen Lyman- $\alpha$ line. The driver pressure was fixed at $0.68 \mathrm{MPa}(100 \mathrm{psi})$, while several tests were performed to optimize the buffer section and diaphragms. Ultimately, a buffer pressure of 0.5 Torr was selected. The buffer/driven diaphragm was a $3 \mu \mathrm{m}$ polyester sheet with thin aluminum coating. The coating was intended to reflect driver radiation, thus preventing radiative heating of the test gas, while the thinness of the diaphragm was necessary to achieve high velocity. The driver/buffer diaphragm was selected as $0.25 \mathrm{~mm}\left(0.010^{\prime \prime}\right)$ Mylar, which gave a slight velocity increase over the more typical 0.30 $\mathrm{mm}(0.012 ")$ aluminum diaphragm. With these settings, the discharge voltages employed were between $21-35 \mathrm{kV}$, depending on driven gas pressure and velocity obtained.

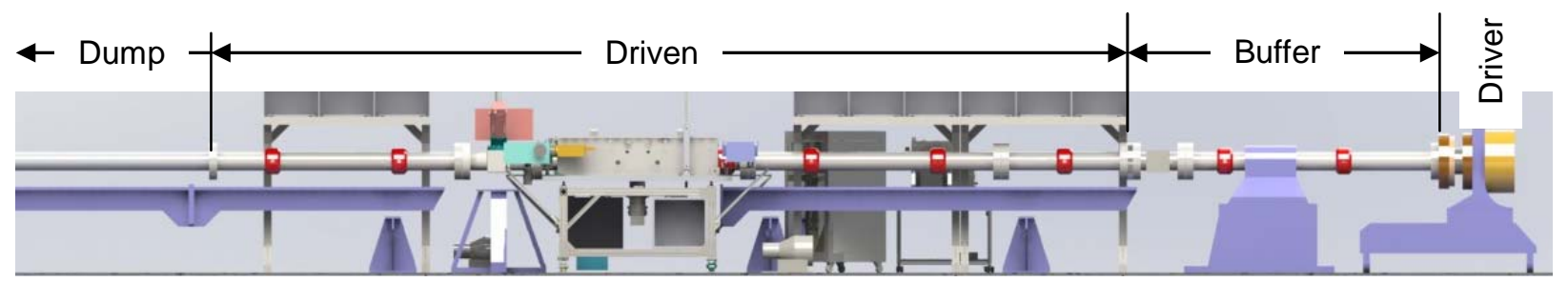

Figure 1. Diagram of the EAST shock tube with driver, buffer and driven sections labeled

The driven tube diameter is $10.16 \mathrm{~cm}$ and the total length of the driven tube, from the main diaphragm to the dump tank, (including the buffer) is $12.5 \mathrm{~m}$. Two turbomolecular pumping stations at either end of the driven tube evacuate the tube to base pressures less than $10^{-5}$ Torr prior to charging with the test gas. The test gas was an 89:11\% mixture of $\mathrm{H}_{2}: \mathrm{He}$ (by volume), which is the approximate composition of Saturn's atmosphere (generally accepted value is from 9.9-13.8\% He [8]. Uranus is estimated to have between 11.9-18.5\% He by volume [9].) The base pressure in the system after 1 minute (about the time required for the test sequence to run) of isolation from the pumps is used to calculate the impurity level for each test. For the tests here, this value was between 40-500 ppm, 
scaling inversely with the test pressure. The test gas and vacuum quality is monitored using a residual gas analyzer from Stanford Research Systems prior to each shot. The optical test section is located approximately $6.75 \mathrm{~m}$ downstream of the main diaphragm. A third diaphragm at the end of the driven tube separates the driven tube from the dump tube and tank.

Along the tube are several round diagnostic ports for time of arrival instrumentation. The ports accommodate flat windows for optical instrumentation or pressure sensors. The shock arrival times are monitored by up to 18 high speed pressure transducers. At a limited number of locations, additional transducers are installed for redundancy and/or planarity measurement. The majority of pressure sensors are model 132A35 piezoelectric shock sensors from PCB electronics. Three of the stations employ the model 113A21 transducer, which has a lower temporal sensitivity but provides more accurate measurements of shock pressure. The times of arrival in the test section are fit with a second-order polynomial, the derivative of which gives shock velocity. For these high velocities, it is found that small errors in the time of arrival detection may have significant impact on the velocity measurement. Therefore the reported velocities may have uncertainty up to $\pm 0.4 \mathrm{~km} / \mathrm{s}$. To monitor radiation from the driver, a $90^{\circ}$ mirror mount was inserted in the center of the shock tube, downstream from the test section. The mirror mount was used to redirect driver (and shock) radiation through the wall window mount, where a photomultiplier tube (PMT) sensitive to $190-320 \mathrm{~nm}$ is mounted. This configuration duplicates that employed in Ref. [5] and its values are compared directly to those obtained in that test series.

Radiation measurement in the EAST is made via 4 spectrometers in two vacuum optical boxes coupled to opposite sides of the test section [10]. The spectrometers image the same axial location in the shock tube through a slot window in the test section. For about half of the tests reported here, fused silica windows were employed, which are transparent between $165-4200 \mathrm{~nm}$. In the other tests, magnesium fluoride $\left(\mathrm{MgF}_{2}\right)$ windows were employed in the vacuum ultraviolet, which are transparent to below $120 \mathrm{~nm}$ and could therefore measure the resonant atomic Lyman- $\alpha$ line and Lyman band radiation from $\mathrm{H}_{2}$. Spectrally and spatially resolved emission from the shocked gas is monitored through these ports. The vacuum box is a rectangular aluminum box which contains all the imaging optics for the EAST spectroscopy implementation. The vacuum box couples to the spectrometers through window ports for ultraviolet (UV), visible (VIS) and near infrared (NIR) spectrometers and through a sealed port for vacuum ultraviolet (VUV) spectrometers. Each vacuum box contains two optical paths, which are each created by a series of six mirrors. The two optical paths enter the test section through the slot windows at slightly different angles, and pass through a focal point at the center axis of the tube. The first two mirrors rotate the image of the slot to a vertical orientation. The remaining mirrors direct the image and focus it onto the spectrometer slits. Each set of mirrors is chosen to optimize particular wavelength ranges depending on the spectrometer they are associated with.

Table I. Spectrometer Settings and Features Observed

\begin{tabular}{|c|c|c|c|c|c|}
\hline $\begin{array}{l}\text { Spectrometer } \\
\text { (Window) }\end{array}$ & $\begin{array}{c}\text { Spectral } \\
\text { Range (nm) }\end{array}$ & Species & Spectral Feature & Transition & $\begin{array}{c}\text { Wavelength } \\
(\mathrm{nm})\end{array}$ \\
\hline \multirow{2}{*}{$\operatorname{VUV}\left(\mathrm{MgF}_{2}\right)$} & \multirow{2}{*}{$117-173$} & $\mathrm{H}$ & Lyman- $\alpha$ & $\mathrm{n}=2 \rightarrow 1$ & 121.57 \\
\hline & & $\mathrm{H}_{2}$ & Lyman Band & $\mathrm{B}^{1} \Sigma-\mathrm{X}^{1} \Sigma$ & $100-160$ \\
\hline VUV $\left(\mathrm{SiO}_{2}\right)$ & $164-218$ & \multicolumn{4}{|c|}{ None } \\
\hline \multirow{6}{*}{ UV } & \multirow{6}{*}{$323-497$} & \multirow{6}{*}{$\mathrm{H}$} & Balmer- $\beta$ & $\mathrm{n}=4 \rightarrow 2$ & 486.14 \\
\hline & & & Balmer- $\gamma$ & $\mathrm{n}=5 \rightarrow 2$ & 434.05 \\
\hline & & & Balmer- $\delta$ & $\mathrm{n}=6 \rightarrow 2$ & 410.17 \\
\hline & & & Balmer- $\varepsilon$ & $\mathrm{n}=7 \rightarrow 2$ & 397.00 \\
\hline & & & Balmer- $\zeta$ & $\mathrm{n}=8 \rightarrow 2$ & 388.91 \\
\hline & & & Balmer Continuum & $\mathrm{n}=\infty \rightarrow 2$ & $<364.6$ \\
\hline Visible & $653-659$ & $\mathrm{H}$ & Balmer- $\alpha$ & $\mathrm{n}=3 \rightarrow 2$ & 656.28 \\
\hline \multirow{2}{*}{ NIR } & \multirow{2}{*}{$1022-1652$} & \multirow{2}{*}{$\mathrm{H}$} & Paschen- $\beta$ & $\mathrm{n}=5 \rightarrow 3$ & 1281.81 \\
\hline & & & Paschen- $\gamma$ & $\mathrm{n}=6 \rightarrow 3$ & 1093.82 \\
\hline
\end{tabular}

The four spectrographs each consist of a CCD array coupled to the focal plane of an imaging spectrometer. They are denoted VUV, UV (or Blue), visible (or Red) and near infrared (NIR). The VUV and Blue spectrometers are coupled to the east side of the shock tube, where the optics box is maintained under high vacuum. The Red and NIR spectrometers are coupled to the west side, but were not maintained under vacuum for this test series. The four spectrometers employed are from PI-Acton and include three $0.5 \mathrm{~m}$ spectrometers (SP2500i) and one $0.39 \mathrm{~m}$ 
vacuum spectrometer (VM504). Each spectrometer has a dedicated computer that operates both the camera and spectrometer simultaneously. Intensity calibration is accomplished using either an integrating sphere $(>300 \mathrm{~nm})$, producing highly uniform output over a $10.2 \mathrm{~cm}$ (4-inch) diameter, or a $\mathrm{D}_{2}$ lamp source $(<300 \mathrm{~nm})$. The $\mathrm{D}_{2}$ lamp source is purged with argon to remove oxygen absorption and mounted to a translating stage to obtain the spectra at different spatial locations. The $\mathrm{D}_{2}$ lamp source is always referenced against the integrating sphere to convert relative intensities to absolute ones. The measurement ranges and features observed for each spectrometer are given in Table I.

\section{B. Computational}

The experimental results are compared to spectra generated by NEQAIR at equilibrium conditions as calculated by the Chemical Equilibrium with Applications (CEA) code [11]. The CEA code is run in incident shock mode and provides species number densities and a temperature that are used as input to NEQAIR [12]. NEQAIR is then run in line of sight mode to give the radiance over a uniform slab of width $D$, where $D$ is the shock tube diameter $(10.16 \mathrm{~cm})$. NEQAIR builds radiative spectra by conducting line-by-line calculations of selected atoms and molecular systems. The current version of NEQAIR (14.0), which has not been fully updated for $\mathrm{H}_{2}$ simulations, contains the NIST v5.0 line list for atomic $\mathrm{H}$ and He and data for simulating the B-X (Lyman), C-X (Werner) and $\mathrm{B}^{\prime}-\mathrm{X}$ bands of $\mathrm{H}_{2}$. $\mathrm{H}$ and He bound-free radiation are calculated based on the TOPBase photoionization rates [13]. The Stark broadening parameters for several $\mathrm{H}$ lines were updated to match those of Gigosos and Cardeñoso [14], though the lines are modeled as a single Lorentzian without fine structure. Quasi-steady state (QSS) rates are not implemented for $\mathrm{H}$ in NEQAIR so that simulations were conducted with Boltzmann distributed state populations. Comparisons to stagnation line results produced through DPLR and NEQAIR reported in our previous work [2] are also given here (Section IV.B), subject to the same limitations in NEQAIR's capability.

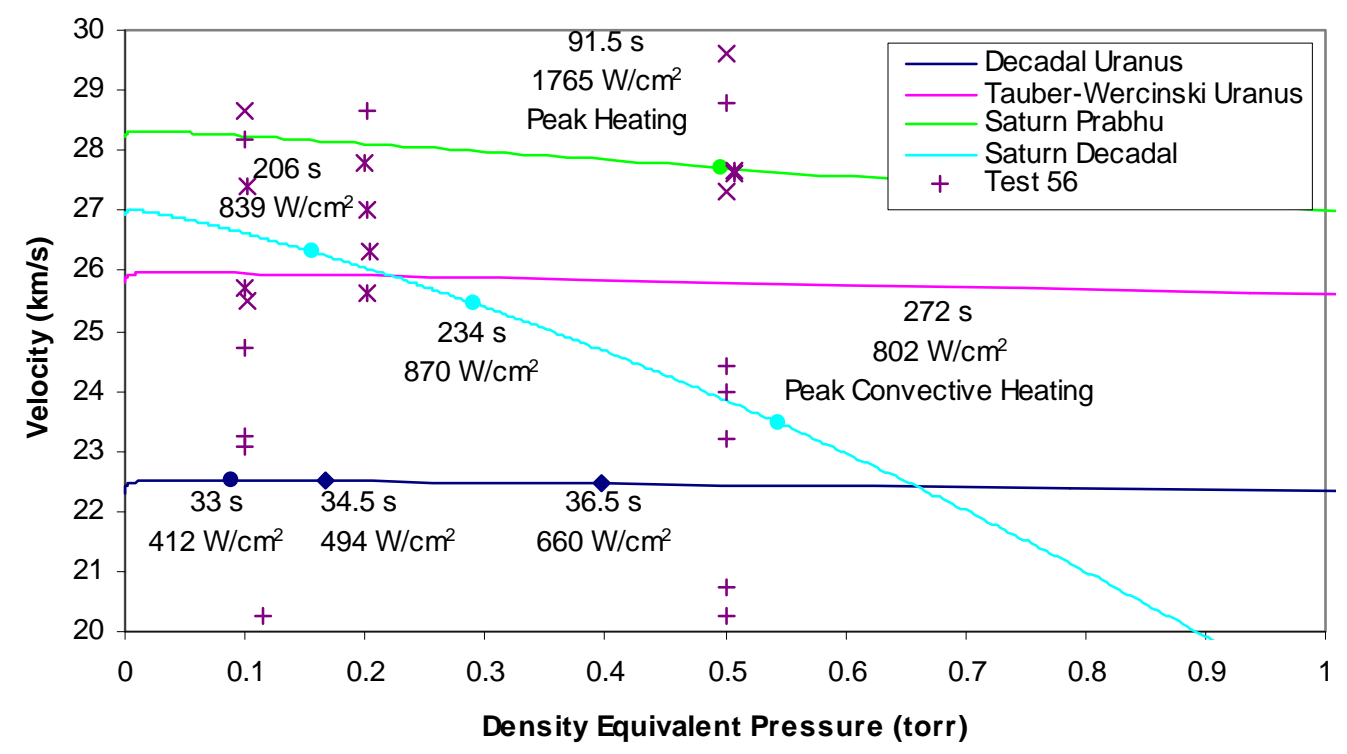

Figure 2. Plots of candidate trajectories for Uranus and Saturn entry overlaid with test conditions performed in this work. The marker style indicates the type of data obtained in the test, as described in the text.

\section{Results}

The test conditions obtained in this work are shown in terms of velocity and freestream (laboratory) pressure, and compared to different proposed Uranus/Saturn trajectories in Figure 2. The shock tube is assumed to have similarity to stagnation line profiles for matched freestream velocity and density. For the trajectories, the atmospheric density has been converted to an equivalent pressure at laboratory conditions. A total of 25 tests were performed (marked with $\mathrm{a}+, \times$ or $*$ in Figure 2) at pressures of 0.1, 0.2 and 0.5 Torr, three of which produced unusable data. Of the remaining 22 tests, 6 had test time but no observable signal. These tests were all less than 25 $\mathrm{km} / \mathrm{s}$, confirming predictions that radiative heating would be negligible at these velocities (which encompass most Uranus trajectories.) From the 16 tests with signal, test times were short or unclear in 4 of them, leaving 12 tests 
with high data quality, marked by an $\times$ in Figure 2. More importantly, 8 of these 12 tests included measurement of the Lyman- $\alpha$ line in the VUV. These tests are marked with a *.

Somewhat surprisingly, no direct evidence of driver gas preheating was seen during any of the tests. Radiance was monitored using the $90^{\circ}$ mirror and compared to those obtained in the test from Ref. [5]. For all tests in this work, radiance from the driver was $15-80 \times$ lower than in the previous work. This is attributed to the blocking affect of the aluminized polyester buffer diaphragm. The shock pressures were always within $20 \%$ of the equilibrium pressure, while in the previous test campaigns they were up to a factor of 3 higher, and the widths of Hydrogen Balmer series lines agreed with equilibrium values to within $20 \%$, whereas before they were an order of magnitude larger. As will be shown below, almost all of the tests were below equilibrium predictions in terms of radiance, temperature, and electron density, suggesting this phenomenon to have been effectively eliminated.

The data in the following is presented in order of ascending velocity. Only selected data sets are shown in this work, but the full set of data can be obtained by contacting the lead author ${ }^{1}$.

\section{A. $20-25 \mathrm{~km} / \mathrm{s}$}

Six shots with valid test time, one at 0.1 Torr and five at 0.5 Torr, were obtained in the range of $20-25 \mathrm{~km} / \mathrm{s}$. None of these displayed any measurable radiation, however the cameras did pick up radiation from the contamination/driver gas. Because of this characteristic, it was initially thought there was no test time at all. By interpolating the camera trigger pulse onto the shock time of arrival curve, it is possible to estimate the location of the shock at the time the camera triggered. The shock would then continue moving during the camera exposure time (generally $0.25 \mu$ s for VUV/Blue/Red cameras and $0.98 \mu$ s for IR.) From this analysis, several centimeters of valid test time with no signal were present on the spectral images. This suggests that any shock radiation present was below the noise floor of the spectrographs. To verify this hypothesis, the equilibrium radiance level is compared to the spectrometer noise floor for several of the shock speeds between $20-25 \mathrm{~km} / \mathrm{s}$ and pressure of 0.5 Torr in Figure 3. The noise floor was identical across all tests, so only one such trace is shown on each figure. For most of these cases, the expected equilibrium level is orders of magnitude less than the noise limit of the spectrometer. Minor exceptions are apparent above $24 \mathrm{~km} / \mathrm{s}$, where the equilibrium prediction of the Balmer series lines at $656,486,434$, and $410 \mathrm{~nm}$ suggests they may be within the detection limits. These lines are not observed here because of a relatively slow equilibration rate in the shock layer, as will be demonstrated in the following sections at higher velocity.
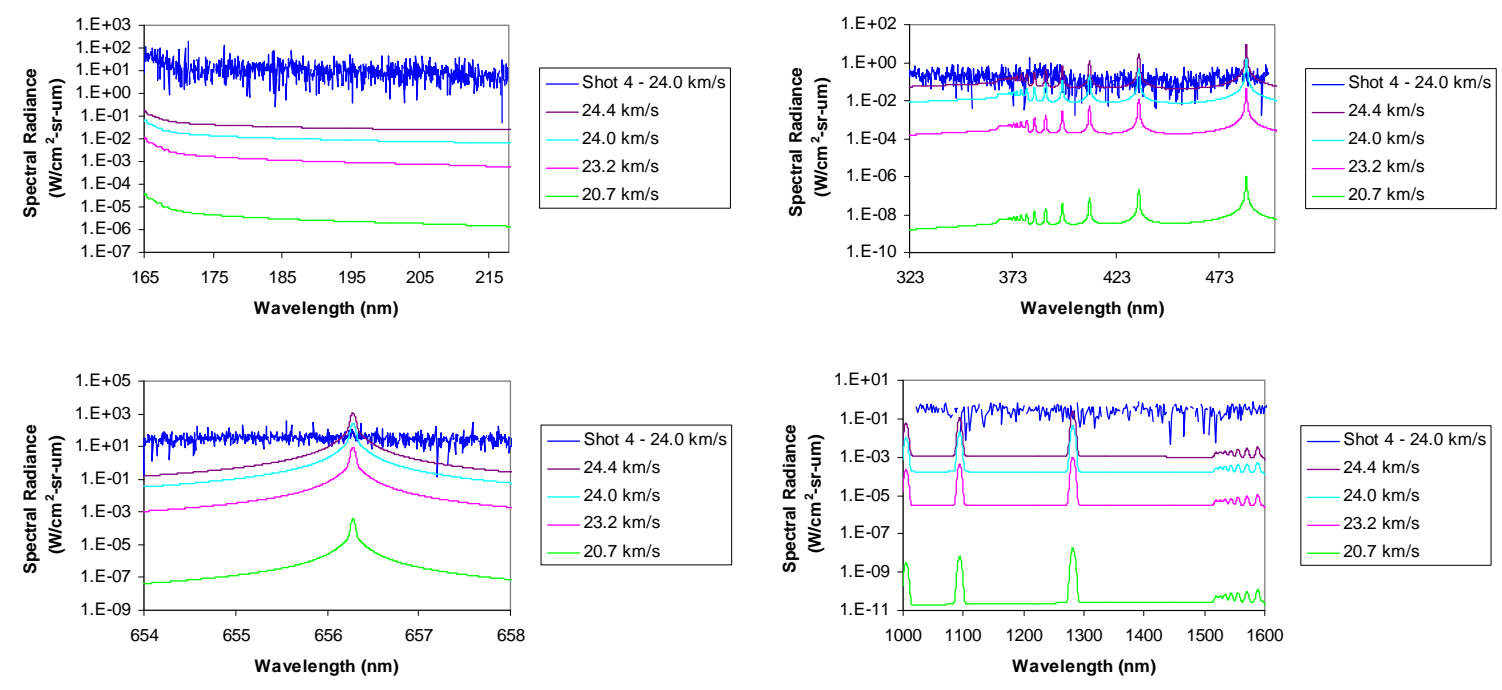

Figure 3. Predicted equilibrium radiances from $20-25 \mathrm{~km} / \mathrm{s}$ compared to the spectrometer noise floor. Because the signal was limited by the noise floor, every test in this velocity range effectively displayed the same signal. Each simulation corresponds to an actual shot condition in the test series.

\footnotetext{
${ }^{1}$ Brett.A.Cruden@nasa.gov
} 

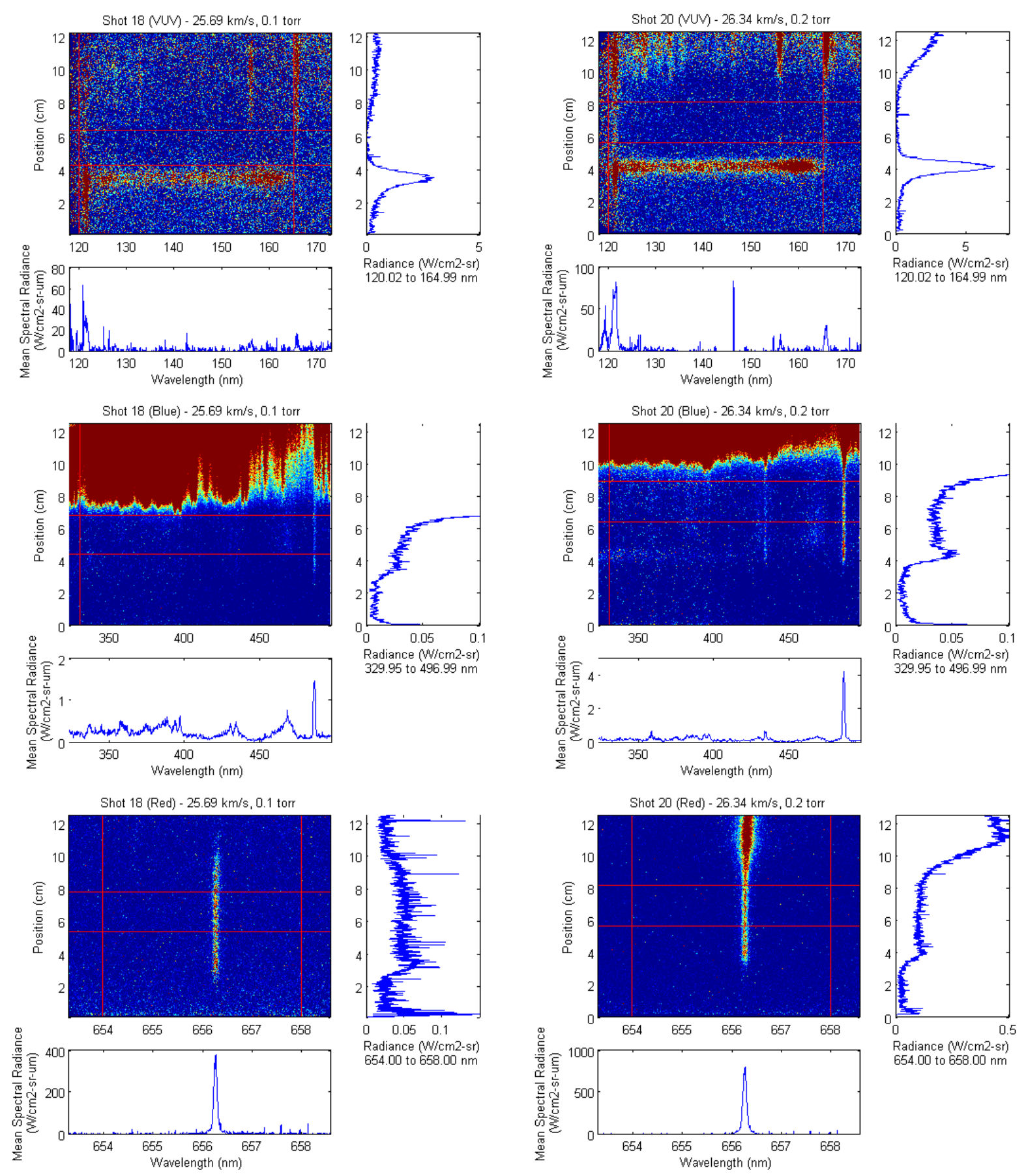

Figure 4. Test data at (left) $25.7 \mathrm{~km} / \mathrm{s}, 0.1$ Torr and (right) $26.3 \mathrm{~km} / \mathrm{s}$ at $0.2 \mathrm{Torr}$. The rows represent the spectral ranges of Deep VUV, UV and Visible.

\section{B. $25-27 \mathrm{~km} / \mathrm{s}$}

There were four shots obtained between $25-27 \mathrm{~km} / \mathrm{s}$, including two at 0.1 Torr and two at 0.2 Torr. A representative data set is given from each of these pressures in Figure 4. The near infrared data is not shown, as any radiance was below the detection limit for these cases. The presence of the shock front is seen with a broad nonequilibrium radiance in the VUV at all conditions, near $y=4 \mathrm{~cm}$. Analysis of the camera trigger and time of arrival shows that this feature actually precedes the shock front. The broad emission is attributed to the Lyman band (B-X) of molecular hydrogen, which is presumably quenched or dissociated when the shock density increases. The 
Lyman- $\alpha$ line at $121.6 \mathrm{~nm}$ is also seen and precedes the shock front by as much as $1-2 \mathrm{~cm}$, with the distance being greater at lower pressure. The nature of the pre-shock radiance will be discussed in Section IV.C. Evidence of Balmer continuum (or possibly molecular) emission is also faintly seen at the shock front on the Blue camera. Clearer evidence of the shock front is ascertained by the appearance of the Balmer- $\alpha, \beta$, and $\gamma$ lines on the Red and Blue cameras. Following the non-equilibrium peak, the radiance settles into a steady level for several $\mathrm{cm}$. The contamination is observed at about $\mathrm{y}=8$ and $10 \mathrm{~cm}$ at 0.1 and 0.2 Torr, respectively. This corresponds to test distances of approximately $4-6 \mathrm{~cm}$.

The radiation intensities from these tests (during the valid test time) are compared against the equilibrium radiance predictions from NEQAIR/CEA for these cases in Figure 5. In the VUV, the only interesting feature expected in equilibrium is the $121.6 \mathrm{~nm}$ Lyman- $\alpha$ line, therefore only the region from 120-130 nm is shown. Both lines are somewhat below equilibrium levels, though the $25.7 \mathrm{~km} / \mathrm{s}$ condition may be considered to be within the noise of the data. The Blue region from $325-500 \mathrm{~nm}$ is shown on a logarithmic scale, as otherwise most features, other than the Balmer- $\beta$ line $(486 \mathrm{~nm}$ ), would be difficult to observe. These features are all significantly less than equilibrium radiation levels, again suggesting that the shock has not equilibrated over the few $\mathrm{cm}$ of test distance. Weak molecular band features near 470,430 and $390 \mathrm{~nm}$ are noted. These may originate from water impurities in the tube $(\mathrm{OH}$ visible $\mathrm{B}-\mathrm{X}$ band) which is not included in the prediction. The Red camera measurement of the Balmer- $\alpha$ line similarly shows significantly lower radiance than expected at equilibrium, with the peaks being 6-7x lower than predicted equilibrium radiance values. There is no measurable infrared signal in these tests, even though the equilibrium levels are above the camera noise floor. The summary of all this data indicates that the steady region behind the shock front is not at an equilibrium condition. It also confirms that significant driver gas radiative heating is not present in these tests.
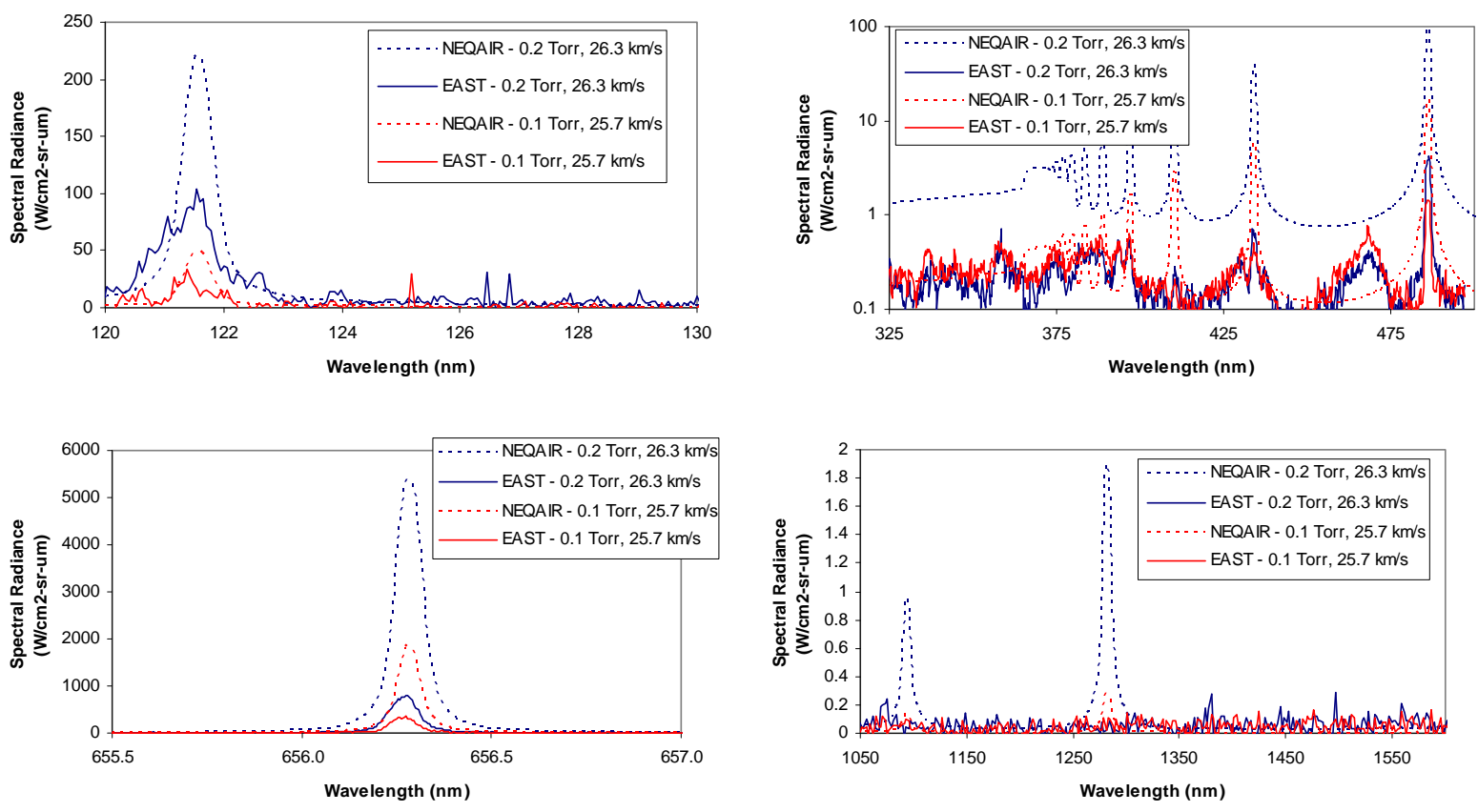

Figure 5. Comparison of EAST spectra to equilibrium radiance levels for velocities of $25.7-26.3 \mathrm{~km} / \mathrm{s}$.

\section{C. $27-28 \mathrm{~km} / \mathrm{s}$}

Six shots were obtained between $27-28 \mathrm{~km} / \mathrm{s}$, including three at 0.5 Torr, two at 0.2 Torr and one at 0.1 Torr. One shot from each of 0.2 Torr and 0.5 Torr conditions are shown in Figure 6. In the 0.2 Torr data (Shot 25), on all 4 cameras, the trigger was such that the shock front is near, or off of, the bottom edge of the CCD array. At 0.5 Torr (Shot 23), the shock front is near $y=4 \mathrm{~cm}$. The Lyman- $\alpha$ line again precedes the molecular emission but not by as much as at the lower pressure conditions. The contact front for Shot 25 occurs near $\mathrm{y}=8 \mathrm{~cm}$ and no contact front is observed in Shot 23. Due to a triggering error in the IR camera during shot 23, no IR data was obtained and the near infrared data from a similar condition (Shot 22) is shown instead. Shot 22 has the shock front at $\mathrm{y}=4 \mathrm{~cm}$ and contact front near $\mathrm{y}=10 \mathrm{~cm}$. A common feature to all these tests is a delayed radiative transition. As at lower velocity, the shock front is observed with molecular emission features in the VUV which then decay to a low value. At around 3- 

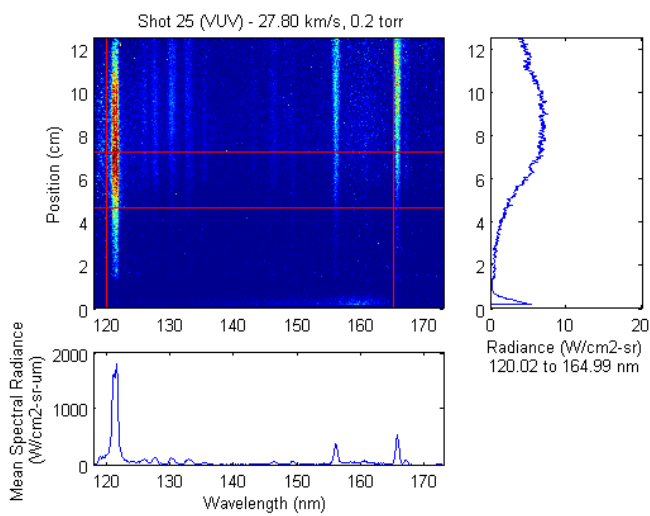

Radiance (W/cm2-sr) 120.02 to $164.99 \mathrm{~nm}$
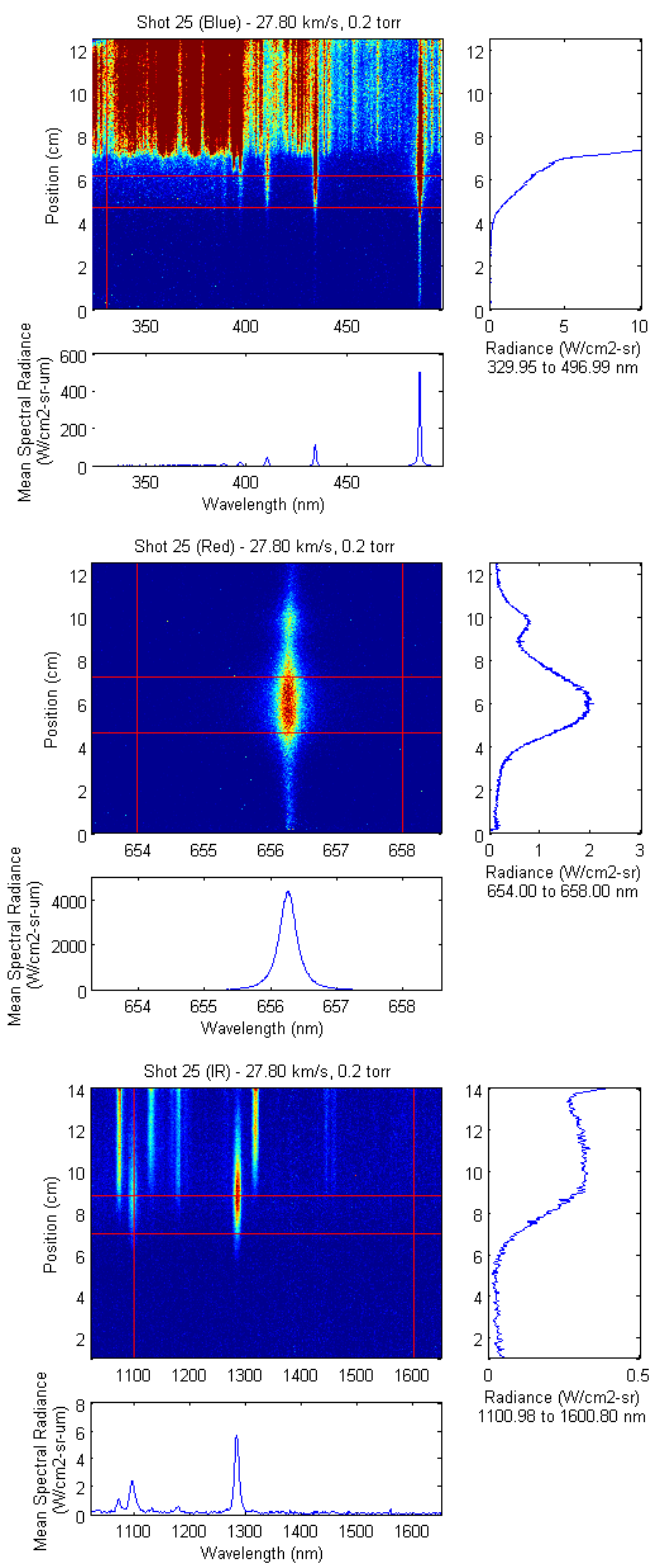
Radiance $(W / \mathrm{cm} 2-\mathrm{sr})$
1100.98 to $1600.80 \mathrm{~nm}$
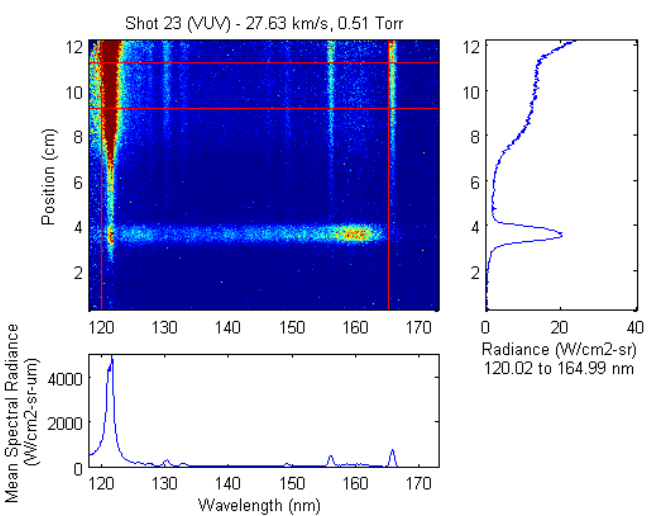

Radiance $(\mathrm{W} / \mathrm{cm} 2-\mathrm{sr})$ 120.02 to $164.99 \mathrm{~nm}$
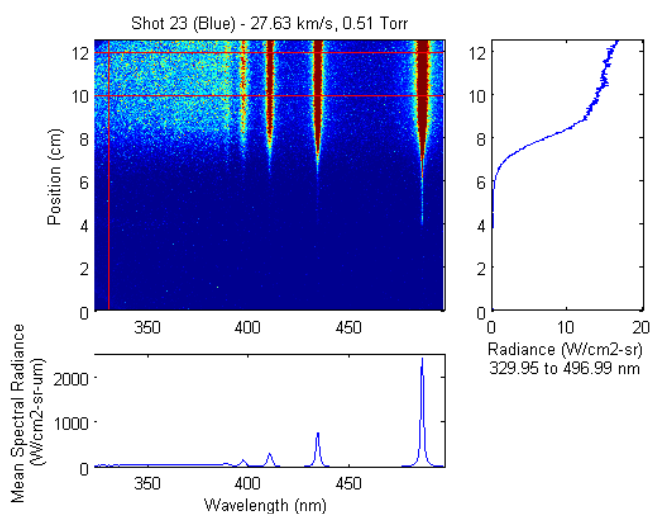

Radiance (W/cm2-sr) 329.95 to $496.99 \mathrm{~nm}$

Shot 23 (Red) - $27.63 \mathrm{~km} / \mathrm{s}, 0.51$ Torr
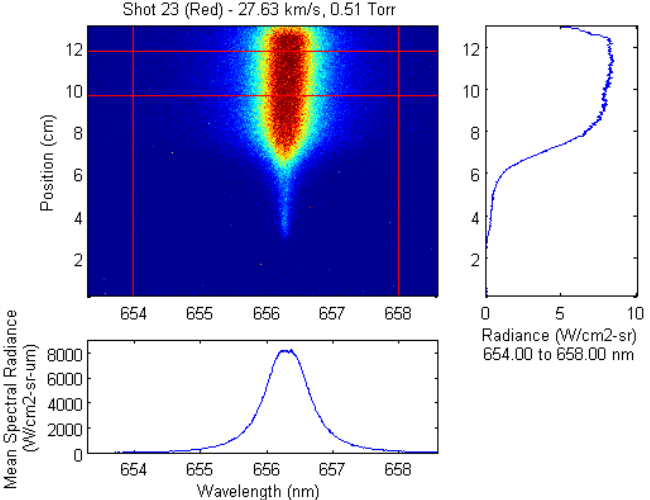

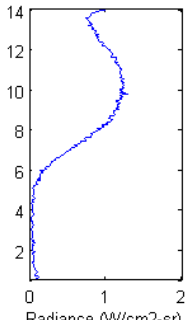

Radiance (W/cm2-sr)
Shot $22(\mathbb{R})-27.66 \mathrm{~km} / \mathrm{s}, 0.51$ tor
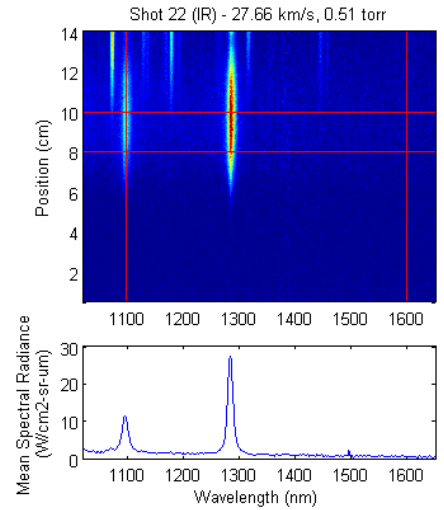

Figure 6. Data collected at (left) between $27.8 \mathrm{~km} / \mathrm{s}$ and 0.2 Torr and (right) $27.6 \mathrm{~km} / \mathrm{s}$ and 0.5 Torr. The rows are ordered by wavelength. 
$4 \mathrm{~cm}$ behind the shock front, the radiation suddenly increases. This trend is observed on all three of the VUV, Blue and Red cameras. The radiation transition is accompanied by an increase in the atomic linewidth. The Balmer- $\alpha$ line at 0.5 Torr displays a flattened top which suggests it is optically thick. In the infrared, no radiation is seen at the shock front. Instead the Paschen- $\beta$ and $\gamma$ lines appear behind the shock front at the same distance from where the radiation spikes on the other cameras. This delayed radiative transition, or induction time, is attributed to a relatively slow ionization rate in the shock layer, and will be discussed in the next section. This transition was consistently observed in this testing regime, though the 0.1 Torr test did not have sufficient test time for this to occur.

The radiance following this transition is compared against equilibrium CEA/NEQAIR calculations in Figure 7. Here, the Lyman- $\alpha$ line exceeds equilibrium for both cases. The Blue camera data is of the same order as the equilibrium computation. At 0.2 Torr, the Balmer- $\beta$ line peak is somewhat above equilibrium and the $\gamma$ line is somewhat below. As lines move to higher energy (lower wavelength), they become increasingly less than equilibrium. At 0.5 Torr, the peak widths are clearly wider in the experiment. Also notable is the absence of any intensity change at about $366 \mathrm{~nm}$, which suggests that the Balmer continuum is not predicted correctly. The Balmer- $\alpha$ line, as measured by the Red camera, is significantly wider in experiment than as given by NEQAIR. This is due to a large optical thickness at a lower effective temperature than equilibrium. The simultaneous observation of a large radiance for the $\mathrm{n}=2 \rightarrow 1$ transition with a lower maximum radiance for the $\mathrm{n}=3 \rightarrow 2$ transition suggests that the $\mathrm{n}=2$ state is overpopulated relative to a Boltzmann distribution. In terms of the total radiative contribution (i.e. area under the curve), the experimental Balmer- $\alpha$ line data is in excess of equilibrium by about $35 \%$ at 0.2 Torr, but $8 \times$ larger at 0.5 Torr. The comparison in the infrared at 0.5 Torr is fairly close to equilibrium, while at 0.2 Torr, the Paschen series radiance in the infrared is slightly less than equilibrium.
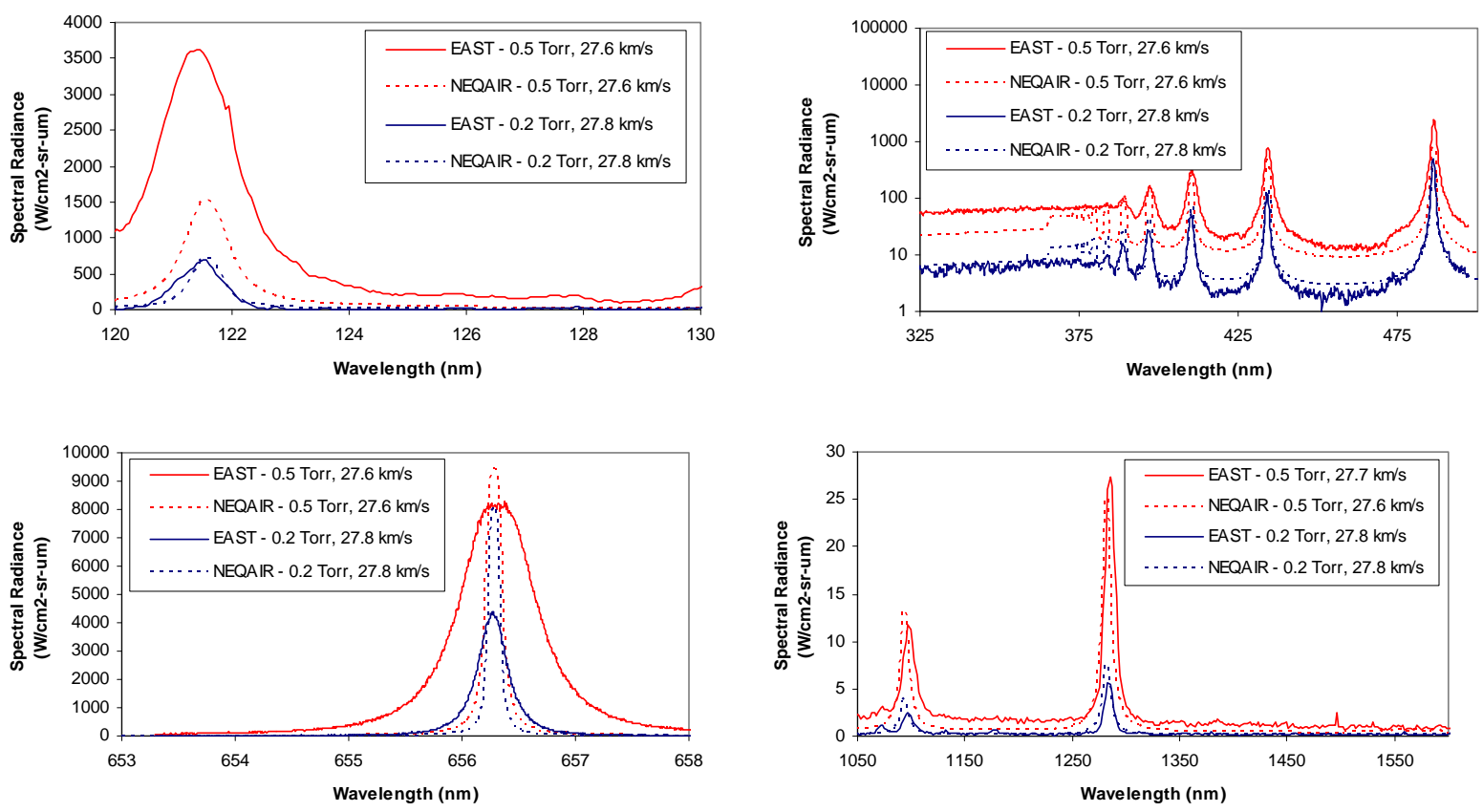

\section{Figure 7. Comparison of $27.6-27.8 \mathrm{~km} / \mathrm{s}$ to equilibrium radiance predictions.}

The 0.2 Torr condition has shown certain lines to exceed equilibrium and others to be less than equilibrium. This trend correlates with the principle quantum number $(\mathrm{n})$ of the state responsible for radiation. In excess of equilibrium are the Lyman- $\alpha(n=2)$, Balmer- $\alpha(n=3)$ and Balmer- $\beta(n=4)$. Below equilibrium are the Balmer- $\gamma$ and higher $(\mathrm{n} \geq 5)$ and the Paschen- $\beta$ and $\gamma(\mathrm{n}=5,6)$. The simplest interpretation of this trend is that the electronic temperature is lower than the equilibrium shock temperature and that the shock is still equilibrating. An alternative, but more complex, interpretation could be that the upper states are being depleted by preferential processes, such as electron impact ionization or radiative decay (non-adiabatic effect). In this case, the state distribution is nonBoltzmann in nature and a state-to-state or QSS model of the hydrogen atom would be required to correctly predict the radiation. 


\section{D. $28-30 \mathrm{~km} / \mathrm{s}$}

Five shots were obtained between $28-30 \mathrm{~km} / \mathrm{s}$, including two at 0.5 Torr, one at 0.2 Torr, and two at 0.1 Torr. Except for one shot at $29.6 \mathrm{~km} / \mathrm{s}$ and 0.5 Torr, these all had short test times. Unfortunately, the deep VUV setting was not measured at $29.6 \mathrm{~km} / \mathrm{s}$, and the Blue camera was saturated. Therefore, these data are not shown. The induction time becomes increasingly shorter at higher velocity and the Balmer- $\alpha$ line more broadened with an extended flat top, again indicative of a blackbody limited emission.

\section{Discussion and Analysis}

\section{A. Electron Density and Temperature Trends}

The spectra may be analyzed for electron density values, as discussed in our earlier work [15]. In the present work, the Balmer- $\alpha$ line has been measured at high resolution, and hence is preferred for Stark analysis. However, since many of the spectra are optically thick, a straight Lorentzian fit of this line may yield unrealistically high electron densities. In this case, the emission is given by:

$$
I=B(\lambda, T)\left[1-\exp \left(-\tau L\left(\frac{\lambda-\lambda_{o}-\lambda_{\text {shift }}}{\Delta \lambda_{1 / 2}}\right)\right)\right]
$$

where $\mathrm{B}(\lambda, \mathrm{T})$ is the blackbody (Planck function) and $\mathrm{L}(\lambda)$ is the Lorentzian function. Fit parameters then include $\mathrm{T}$, $\tau, \lambda_{\text {shift }}$, and $\Delta \lambda_{1 / 2}$. For the optically thick case, the fit is fairly insensitive to electron density, while it is insensitive to temperature for the optically thin case. Therefore only temperature or electron density can be reliably reported for a single line. The fit must include a convolution with the instrument lineshape, and the sensitivity limit of the measurement will depend on how close the linewidth is to the instrument lineshape. The high resolution lineshape used for the $\alpha$ line is equivalent to an electron density between $2-4 \times 10^{14} \mathrm{~cm}^{-3}$, depending on the slit settings used. The $\beta$ and $\gamma$ lines, being measured at low resolution, had widths corresponding to of $5.7-9.2 \times 10^{15}$ and $4.0-6.5 \times 10^{15}$ $\mathrm{cm}^{-3}$, respectively. Depending on the quality of the signal, it may be possible to detect densities lower than these values, as the line broadening is additive, to first order. The temperature, when fit, is the equivalent temperature between the $n=2$ and 3 states and is only equal to the electronic temperature if the states follow a Boltzmann distribution.

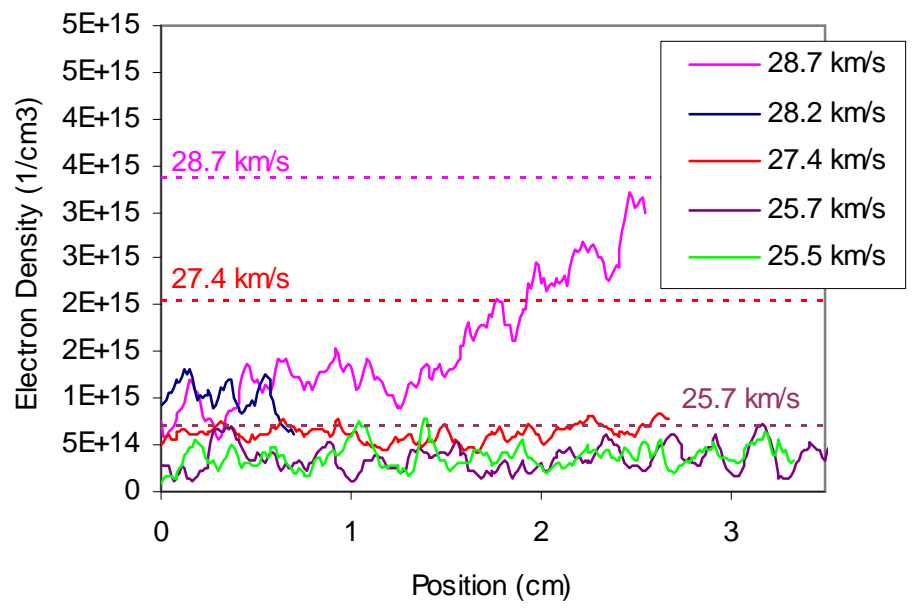

Figure 8. Electron Density deduced from Balmer- $\alpha$ linewidth at pressure of 0.1 Torr. Dotted lines display equilibrium electron densities at 28.7, 27.5 and $25.7 \mathrm{~km} / \mathrm{s}$. (Equilibrium densities at 28.2 and $25.5 \mathrm{~km} / \mathrm{s}$ are omitted for clarity)

The 0.1 Torr tests are all optically thin, so electron density is obtained from the $\alpha$ line without temperature data. The $\beta$ and $\gamma$ line measurements are limited by instrument sensitivity and thus not shown. The plots in Figure 8 have been shifted so that the shock front (which is known to within $\sim 0.5 \mathrm{~cm}$ ) is at $\mathrm{x}=0$, and the traces have been truncated at the end of the test time. The dotted lines display equilibrium electron densities at $28.7,27.4$ and $25.7 \mathrm{~km} / \mathrm{s}$. Of the five traces shown, all but the $28.7 \mathrm{~km} / \mathrm{s}$ case are flat over the course of the test and are near the instrument 
linewidth value. Therefore, it is possible the actual electron density is lower than these curves suggest. The electron density at $28.7 \mathrm{~km} / \mathrm{s}$ begins to increase noticeably approximately $1.3 \mathrm{~cm}$ after the shock front. From here it continues increasing until the end of the test approximately $2.5 \mathrm{~cm}$ behind the shock front. At this point, it is nearing the equilibrium electron density but is still lower than equilibrium. This is consistent with emission observations, which indicate the shock is not yet in equilibrium.
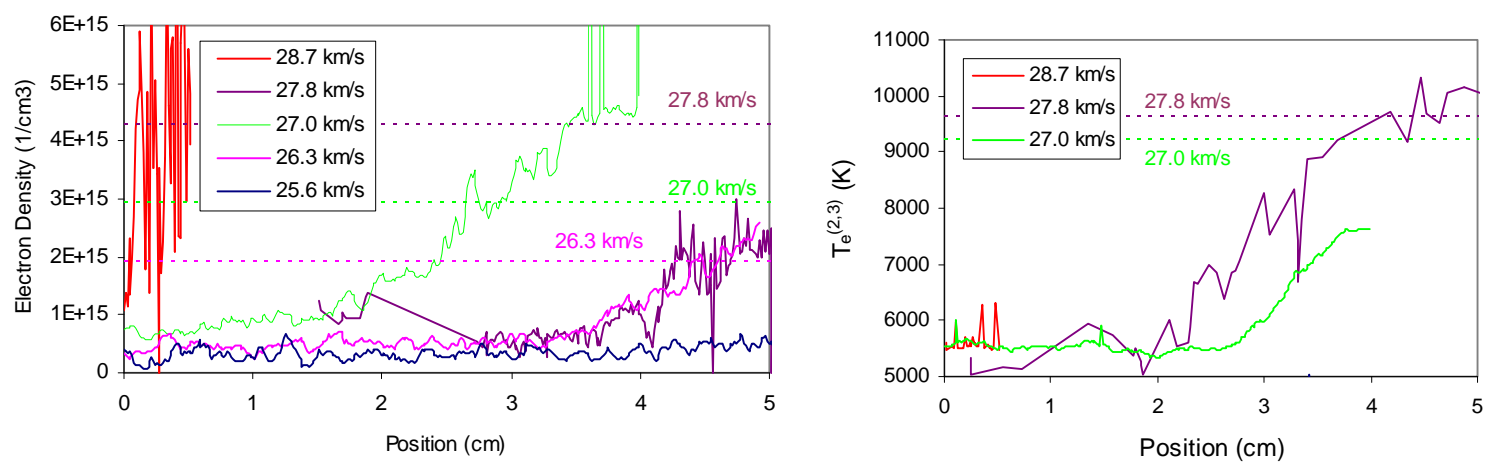

Figure 9. (a) Electron Density and (b) Temperature deduced from $\mathrm{H}-\alpha$ and/or $\gamma$ linewidth and intensity at pressure of 0.2 Torr. Dotted lines display equilibrium values at $27.8,27.0$ and $26.3 \mathrm{~km} / \mathrm{s}$.

At 0.2 Torr, optical thickness is only observed above $27 \mathrm{~km} / \mathrm{s}$. Therefore, temperature plots are given for only three of the velocities. The $28.7 \mathrm{~km} / \mathrm{s}$ case has a test time of about $0.2 \mu \mathrm{s}$, so that only a few points are given. The $27.8 \mathrm{~km} / \mathrm{s}$ case does not begin increasing in electronic temperature until about $1.4 \mathrm{~cm}$ behind the shock front. From here it increases until it has reached equilibrium near the end of the test time. At $27.0 \mathrm{~km} / \mathrm{s}$, the shock becomes blackbody limited at approximately $3 \mathrm{~cm}$ behind the shock front. Before this point, it is not entirely clear from the data whether it is blackbody limited or not. The temperature presented then may be an upper bound of the actual electronic temperature. The temperature increases monotonically from $3-4 \mathrm{~cm}$ behind the shock front where the test time ends, still several thousand degrees below equilibrium. The electron densities are shown in Figure 9(b). Here, the $\alpha$ line cannot be used above $27 \mathrm{~km} / \mathrm{s}$ due to the optical thickness. At $27.8 \mathrm{~km} / \mathrm{s}$, the density from the $\gamma$ line is shown. The electron density stays low until about $3 \mathrm{~cm}$ behind the shock where it begins increasing. The density is still below equilibrium at the end of the test time. At $27 \mathrm{~km} / \mathrm{s}$, the $\alpha$ line density is shown until $3 \mathrm{~cm}$ from the shock front where it switches to the $\beta$ line. The $27.0 \mathrm{~km} / \mathrm{s}$ data appears inconsistent with the rest, starting to increase near $2 \mathrm{~cm}$ and rising above equilibrium at $3 \mathrm{~cm}$. This inconsistency may be due to a relatively higher base pressure/impurity level in this particular test. At the two lowest velocities, the density obtained from the $\alpha$ line is given. The $26.3 \mathrm{~km} / \mathrm{s}$ data follows the same trend as at $27.8 \mathrm{~km} / \mathrm{s}$, beginning to increase at $3 \mathrm{~cm}$. By the end of the test, it is at the equilibrium level. At $25.6 \mathrm{~km} / \mathrm{s}$, the electron density is near the detection limit the whole test and does not increase over the entire $5 \mathrm{~cm}$.

At 0.5 Torr, all cases are blackbody limited. Temperatures are obtained from the $\alpha$ line while electron densities are found from the $\gamma$ lines. The $\beta$ line density is generally $1.6-1.9 \times$ lower, which is attributed to the electron density being too near the instrument linewidth. The curves are shown in Figure 10 and have been shifted so as to place $\mathrm{x}=0$ at the estimated shock front location. The temperature trends appear as a series of $\mathrm{S}$ curves, with the delay time increasing at lower shock velocity. The temperature is found to ramp from approximately $5500 \mathrm{~K}$ to $8000 \mathrm{~K}$ fairly quickly, and continues rising slowly after that. In none of these cases does the temperature reach the equilibrium level. While most of these cases have less than $10 \mathrm{~cm}$ of test time, the temperature curve is well behaved, so is carried out that far for most of the plots. The electron density curves are shown only for valid test time in Figure 10(b). Electron density was not obtained at $29.6 \mathrm{~km} / \mathrm{s}$ due to camera saturation. At $28.8 \mathrm{~km} / \mathrm{s}$, the electron density is measured near $9 \times 10^{15} \mathrm{~cm}^{-3}$ during its short test time. At $27.6-27.7 \mathrm{~km} / \mathrm{s}$, it takes about $2 \mathrm{~cm}$ before electron density becomes reliably detected. The density continues to rise following this, and is near equilibrium levels at 8 $\mathrm{cm}$ beyond the shock front. At $27.3 \mathrm{~km} / \mathrm{s}$, the electron density is first detected near $4 \mathrm{~cm}$, and continues rising, but is still far from equilibrium at the end of the test almost $6 \mathrm{~cm}$ behind the shock front. It is noteworthy that the electron density is first observed approximately where the temperature begins to plateau. This makes sense because a high electron temperature will be required to drive ionization. As the electron density rises, however, the temperature of the shock should fall, which is contrary to the measured temperature trend. This inconsistency indicates that the 
electronic temperature has not equilibrated to the translational temperature. It may also be the case that the hydrogen state population has not yet attained a Boltzmann distribution.
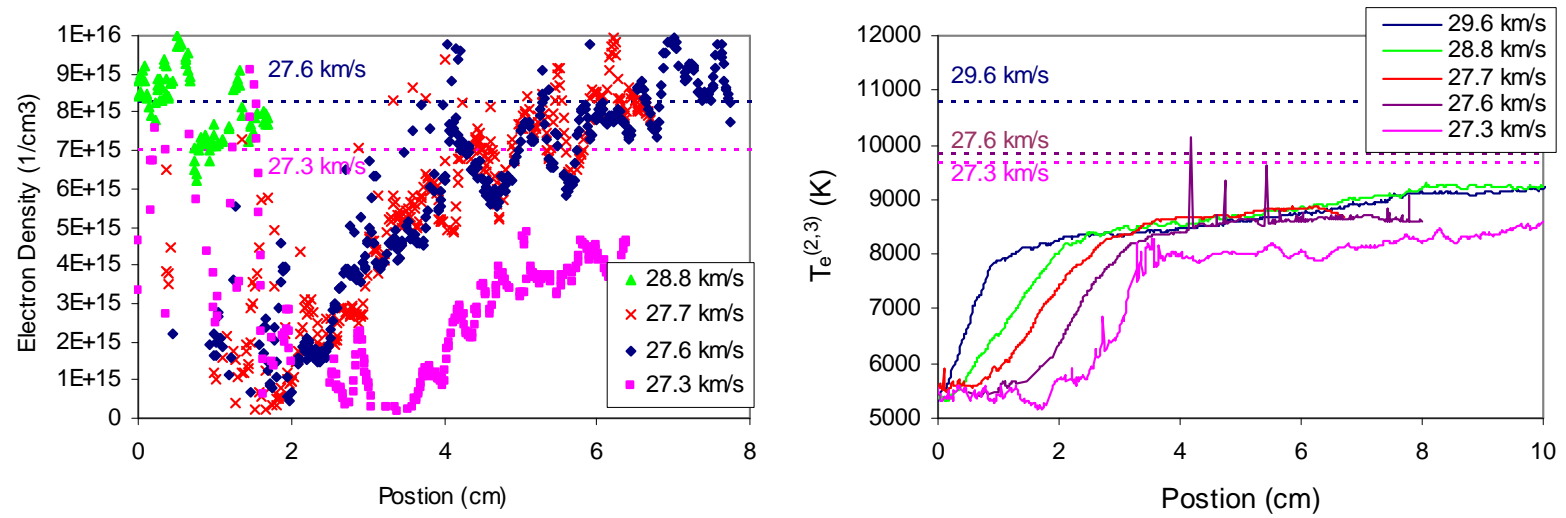

Figure 10. (a) Electron densities and (b) electronic temperature estimated from Hydrogen Balmer series lines at 0.5 Torr. Equilibrium temperature/densities are shown as dotted lines at 27.3, 27.6 and $29.6 \mathrm{~km} / \mathrm{s}$.

\section{B. Comparison to flight CFD}

Since the predictions for Saturn probe entry generally have shock stand-off distances between 2-2.5 $\mathrm{cm}[2]$, the EAST experiment may be considered to replicate the entire stagnation line. Two relevant points from the uncertainty study were obtained for a velocity of $27.7 \mathrm{~km} / \mathrm{s}$ and density equivalent pressure of 0.49 Torr and 26.3 $\mathrm{km} / \mathrm{s}$ and 0.15 Torr. Shot 23 will be compared to the first case, while Shot 20 will be compared to the second.
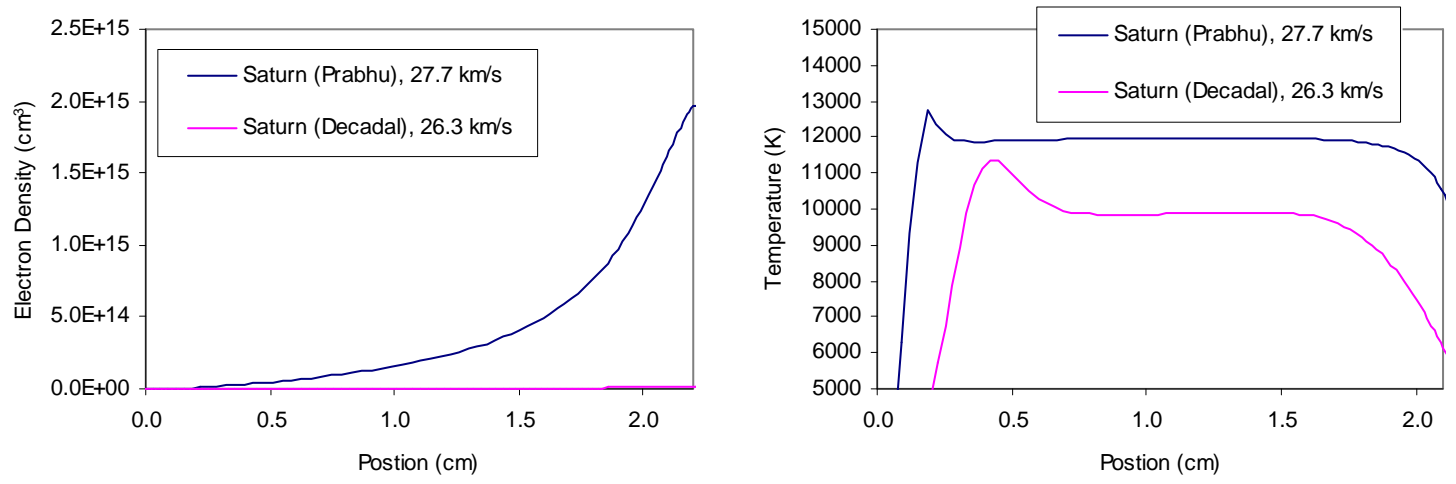

Figure 11. Stagnation line predictions [2] corresponding to the $91.5 \mathrm{~s}$ point on the Prabhu (-15 ${ }^{\circ}$ entry) trajectory and $206 \mathrm{~s}$ on the Decadal Survey $\left(-8^{\circ}\right.$ entry) trajectory. (a) Electron density, (b) Vibrational temperature.

Stagnation line predictions of electron density and vibrational temperature (assumed equal to electron/electronic temperature) are shown in Figure 11. The shock front, defined as when pressure reaches 1\% of its equilibrium value, is placed at the origin. For the $27.7 \mathrm{~km} / \mathrm{s}$ condition, the CFD simulation predicts the electron density exceeding $10^{15} \mathrm{~cm}^{-3}$ at about $2 \mathrm{~cm}$ beyond the shock front, which is in general agreement with the shock tube data. The $26.3 \mathrm{~km} / \mathrm{s}$ obtains a maximum electron density near $10^{13} \mathrm{~cm}^{-3}$ after $2 \mathrm{~cm}$, which would not be detected in the shock tube. The ionization levels at the shock front correspond to fractions near $10^{-4}$, while the equilibrium levels are closer to $10^{-2}$. The impact of impurities on the kinetics then cannot be discounted -- a base pressure above $10^{-5}$ torr means that the impurity fraction (mostly water vapor, but also $\mathrm{N}_{2}, \mathrm{O}_{2}$ and/or $\mathrm{CO}_{2}$ ) is comparable to the ionization fraction. Given that all of these species are more readily ionized than atomic $\mathrm{H}$ means they may alter the electron density and, hence, reaction rates.

The CFD temperature is predicted to rise to $12,000 \mathrm{~K}$ within a few millimeters of the shock front at the higher pressure condition. For the lower velocity/pressure case, a steady state temperature is obtained within about $7 \mathrm{~mm}$. 
In both cases, later in the shock, the temperature begins to decrease as ionization becomes significant. This effect is obscured, however, by the presence of the boundary layer in the flight simulation.
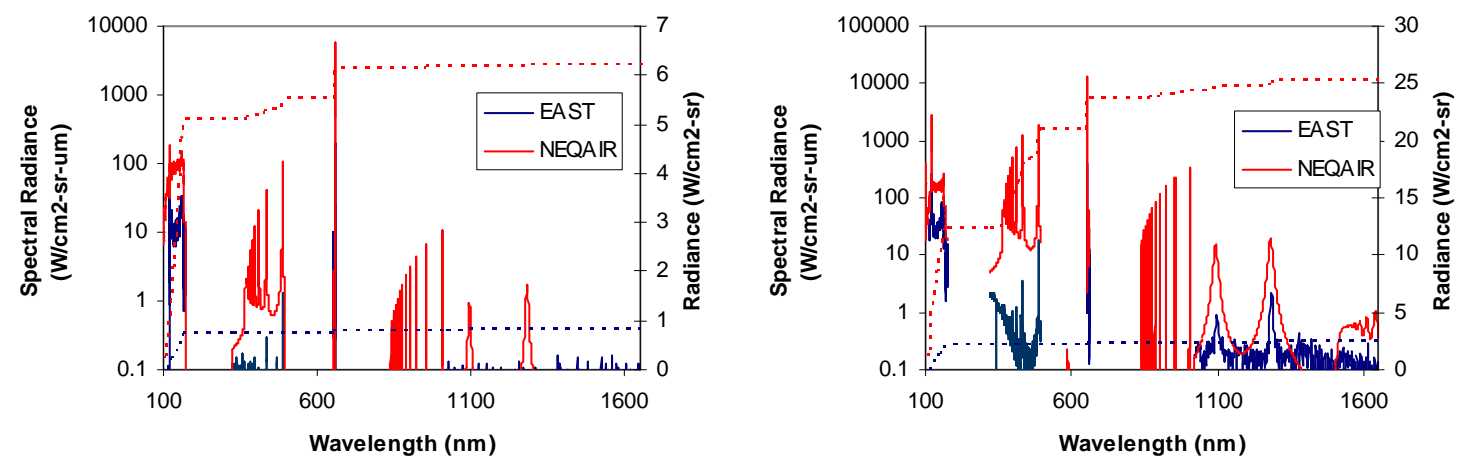

Figure 12. Comparison of EAST estimates of stagnation line radiance and DPLR/NEQAIR predicted radiances for (a) Shallow (-8 $)$ Saturn Entry Trajectory and (b) Steep $\left(-15^{\circ}\right)$ Saturn Entry Trajectory. The solid lines display the spectral radiance (primary axis) and the dashed lines are the accumulated radiance over measured spectral ranges (secondary axis).

As a final test of the predictive capability, the shock tube data is normalized by the shock tube width and integrated in space to obtain an estimated radiative heating profile. The integration is performed over $2.5 \mathrm{~cm}$ for shot 23 and $2.0 \mathrm{~cm}$ for shot 20 , to be consistent with predicted shock stand-off distances. This integrated quantity can then be compared to the stagnation line of sight produced by NEQAIR. This comparison is shown in Figure 12. While the major features are present in the ranges measured, the magnitude is substantially overpredicted. Primarily, this results from having the non-Boltzmann distribution as suggested above. Considering the radiance from the measured regions only, which contributes $\sim 90 \%$ of the predicted radiance, the radiance is only about 2.5 $\mathrm{W} / \mathrm{cm}^{2}$-sr and $0.9 \mathrm{~W} / \mathrm{cm}^{2}$-sr for shot $23 / 22$ and 20 , respectively. In terms of radiative heating, this would contribute at most $16 \mathrm{~W} / \mathrm{cm}^{2}$ and $5.4 \mathrm{~W} / \mathrm{cm}^{2}$, compared to the nominal (i.e. DPLR/NEQAIR) predicted heating of $133 \mathrm{~W} / \mathrm{cm}^{2}$ and $34 \mathrm{~W} / \mathrm{cm}^{2}$. This result is summarized in Table II. However, the approximations made thus far are only valid for an optically thin flow. Features from the VUV and Red cameras are at least partially optically thick, which could increase the radiative heating by several times over this rough calculation. However, the NEQAIR result will still be an overestimate of the actual heating. Implementation of a QSS model should reduce the radiative prediction significantly and bring it closer in-line with observations.

Table II. Estimated Stagnation Line Radiative Heat Flux for a 1-m diameter Saturn probe from predictive simulations and shock tube tests

\begin{tabular}{|c|c|c|c|c|c|}
\hline \multicolumn{3}{|c|}{ DPLR/NEQAIR } & \multicolumn{3}{c|}{ EAST Inferred } \\
\hline $\begin{array}{c}\text { Shock } \\
\text { Velocity } \\
(\mathrm{km} / \mathrm{s})\end{array}$ & $\begin{array}{c}\text { Freestream } \\
\begin{array}{c}\text { Density } \\
\left(\mathrm{kg} / \mathrm{m}^{3}\right)\end{array}\end{array}$ & $\begin{array}{c}\text { Radiative } \\
\text { Heat Flux } \\
\left(\mathrm{W} / \mathrm{cm}^{2}\right)\end{array}$ & $\begin{array}{c}\text { Shock } \\
\text { Velocity } \\
(\mathrm{km} / \mathrm{s})\end{array}$ & $\begin{array}{c}\text { Freestream } \\
\text { Density } \\
\left(\mathrm{kg} / \mathrm{m}^{3}\right)\end{array}$ & $\begin{array}{c}\text { Radiative } \\
\text { Heat Flux } \\
\left(\mathrm{W} / \mathrm{cm}^{2}\right)\end{array}$ \\
\hline 26.3 & $1.8 \times 10^{-5}$ & 34 & 26.3 & $2.4 \times 10^{-5}$ & 5.4 \\
\hline 27.7 & $5.8 \times 10^{-5}$ & 133 & 27.6 & $5.9 \times 10^{-5}$ & 16 \\
\hline
\end{tabular}

It is of interest to compare these results to those of Park [16] and Hollis [4] for Neptune entries. The Neptune entry condition is generally at higher density and velocity than studied in this work, however the $\mathrm{t}=210$ s point lies between the test conditions at 0.5 Torr, having a density of $5.9 \times 10^{-5} \mathrm{~kg} / \mathrm{m}^{3}$ and velocity of $25.73 \mathrm{~km} / \mathrm{s}$. In these works, radiative heating between of 75 and $130 \mathrm{~W} / \mathrm{cm}^{2}$ were reported for a shock standoff distance of $2.8 \mathrm{~cm}$. Interpolation of Park's Table 4 would produce heat fluxes of 80 and $800 \mathrm{~W} / \mathrm{cm}^{2}$ for the conditions reported in Table II. The difference here is presumably due to the presence of $1.5 \% \mathrm{CH}_{4}$ in the Neptune simulations which increases equilibration rates and introduces additional radiating species, thereby increasing the radiation by several times over that in the absence of hydrocarbons. The upper atmosphere of Uranus is understood to have less than $10^{-6}$ fraction of Methane above $200 \mathrm{~km}$ altitude. [17] Saturn, however, may be as high as $0.45 \% \mathrm{CH}_{4}$ at altitudes of interest[18], so that this effect warrants further study for Saturn entries. (Note that the $\mathrm{CH}_{4}$ concentration in Neptune at altitudes relevant for entry is near $0.6 \%$ [19]). 


\section{Pre-shock Excitation}

Pre-shock excitation is observed in the vacuum ultraviolet for velocities greater than $25 \mathrm{~km} / \mathrm{s}$. (Conditions lower than $25 \mathrm{~km} / \mathrm{s}$ were not measured in the far vacuum ultraviolet). This includes radiation from the Lyman bands of $\mathrm{H}_{2}$ and the Lyman- $\alpha$ line of $H$. Evidence of the pre-shock radiance is also apparent in the Balmer series. Park has recently proposed that low levels of driver radiation can excite the pre-shock region, thereby elevating the freestream energy and altering the final condition of the shocked gas [20]. In his study, he predicted that absorption of a $16,000 \mathrm{~K}$ blackbody could cause the atomic hydrogen concentration to increase up to $0.2 \%$ by mole and the vibrational/electronic/electron temperature to rise up to $14,000 \mathrm{~K}$ in the freestream. Though the tests studied in that work were at higher pressure and velocity than the present study, it is qualitatively consistent with the pre-shock radiance observed here. However, at the same time, the heated gas may be expected to diffuse ahead of the shock front, resulting in both elevated temperatures and mole fractions in the pre-shock region. The CFD solution of Figure 11, for instance, had the peak translational temperature occurring before the shock reaches a steady pressure. The domain boundary, being only a few $\mathrm{mm}$ in front of the shock, will limit the amount of diffusion allowed in the prediction. The pre-shock regions may therefore be as hot as several thousands of degrees.

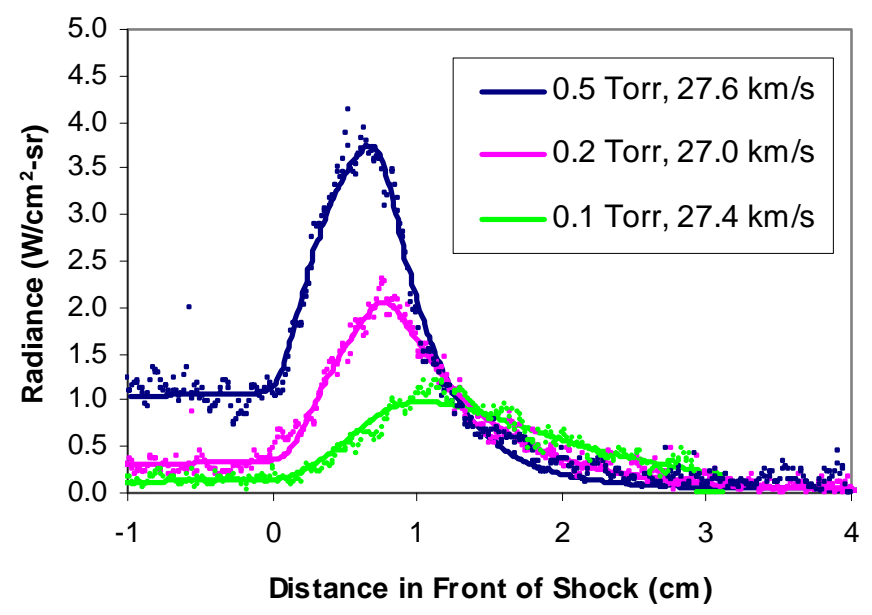

Figure 13. Radiance observed in front of the shock attributable to the Lyman- $\alpha$ line $(120-128 \mathrm{~nm})$ at three pressures. The solid lines are fit exponential rise and decays, convolved with the spatial resolution function, while the points are the data.

The rise and decay in free-stream radiance have been fit with exponential functions to determine their characteristic lengths. To properly perform the analysis, the exponential function is convolved with the spatial resolution function of the test. [21] Sample fits are shown in Figure 13 and the corresponding relaxation coefficients are given in Table III. Also given in Table III are the equivalent diffusion or photoexcitation rates to match the decay coefficient. These rates are based on a 1-D model of the pre-shock region. For photoexcitation, the radiance is given by:

$$
\frac{d I}{d x}=-\sigma_{e x} n_{H_{2}} I
$$

which has a solution given as:

$$
I=I_{0} e^{-\sigma_{e x} n_{H_{2}} x}
$$

so that the characteristic length is given as $1 / \sigma_{\mathrm{ex}} \mathrm{n}$. The cross-section $\sigma_{\mathrm{ex}}$ will correspond to photodissociation (for H$\alpha$ emission) or photoexcitation (for B-X emission). The equivalent excitation cross-sections are near $2 \times 10^{-16} \mathrm{~cm}^{2}$ for $\mathrm{H}-\alpha$ and from $4-6 \times 10^{-16} \mathrm{~cm}^{2}$ for $\mathrm{H}_{2}$. These decay rates are faster than expected for $\mathrm{H}_{2}$ absorption processes, which peak at $10^{-17} \mathrm{~cm}^{2}$ for dissociation and $10^{-16} \mathrm{~cm}^{2}$ for excitation.[20]

The diffusion rates are derived from the 1-D drift-diffusion equation:

which has solution given as:

$$
v \frac{d n_{H}}{d x}=-D_{H} \frac{d^{2} n_{H}}{d^{2} x}
$$




$$
n_{H}=n_{H}^{0} e^{-\frac{v x}{D_{H}}}
$$

so that the characteristic length is $D_{H} / v$. For this case the equivalent diffusion coefficients are between $80-300 \mathrm{~m}^{2} / \mathrm{s}$ for $\mathrm{H}$ and $40-130 \mathrm{~m}^{2} / \mathrm{s}$ for $\mathrm{H}_{2}$. These values match the diffusivity of $\mathrm{H}$ and self-diffusivity of $\mathrm{H}_{2}$ in the freestream mixture for temperatures between about 4000-6000 K. Also shown in Table III is the mean free path calculated for a hot hydrogen atom or molecule entering the cold free-stream. The characteristic lengths are on average 2 mean free paths, therefore it is plausible that pre-shock temperatures may be this large. The rise times obtained in front of shock are not reported in Table III. These characteristic lengths are generally faster than the decay and are often the same order as, or less than, the mean free path.

Table III. Analysis of pre-shock decay length and deduced diffusivity or excitation/dissociation cross-sections

\begin{tabular}{|c|c|c|c|c|c|c|c|c|c|}
\hline \multirow{2}{*}{$\begin{array}{c}\text { Pressure } \\
\text { (torr) }\end{array}$} & \multirow{2}{*}{$\begin{array}{l}\text { Velocity } \\
(\mathrm{km} / \mathrm{s})\end{array}$} & \multicolumn{4}{|c|}{$H-\alpha$} & \multicolumn{4}{|c|}{$\mathrm{H}_{2}(\mathrm{~B}-\mathrm{X})$} \\
\hline & & $\begin{array}{l}\text { Decay } \\
\text { Length } \\
\text { (cm) }\end{array}$ & $\begin{array}{c}\mathrm{D}_{\mathrm{H}} \\
\left(\mathrm{m}^{2} / \mathrm{s}\right)\end{array}$ & $\sigma_{\text {diss }}\left(\mathrm{cm}^{2}\right)$ & $\begin{array}{l}\lambda_{\text {mfp }} \\
(\mathrm{cm})\end{array}$ & $\begin{array}{c}\text { Decay } \\
\text { Length } \\
(\mathrm{cm})\end{array}$ & $\begin{array}{c}\mathrm{D}_{\mathrm{H} 2} \\
\left(\mathrm{~m}^{2} / \mathrm{s}\right)\end{array}$ & $\sigma_{\text {exc }}\left(\mathrm{cm}^{2}\right)$ & $\begin{array}{l}\lambda_{\mathrm{mfp}} \\
(\mathrm{cm})\end{array}$ \\
\hline \multirow[t]{2}{*}{0.1} & 25.7 & 1.03 & 264 & $3.0 \times 10^{-16}$ & \multirow[t]{2}{*}{0.66} & 0.50 & 128 & $6.2 \times 10^{-16}$ & \multirow[t]{2}{*}{0.25} \\
\hline & 27.4 & 0.80 & 219 & $3.8 \times 10^{-16}$ & & 0.44 & 122 & $6.9 \times 10^{-16}$ & \\
\hline \multirow[t]{3}{*}{0.2} & 25.6 & 0.51 & 132 & $3.0 \times 10^{-16}$ & \multirow{3}{*}{0.33} & 0.17 & 45 & $8.8 \times 10^{-16}$ & \multirow[t]{3}{*}{0.12} \\
\hline & 26.3 & 0.69 & 181 & $2.2 \times 10^{-16}$ & & 0.26 & 70 & $5.8 \times 10^{-16}$ & \\
\hline & 27.0 & 0.61 & 164 & $2.5 \times 10^{-16}$ & & 0.26 & 70 & $6.0 \times 10^{-16}$ & \\
\hline \multirow[t]{2}{*}{0.5} & 27.6 & 0.31 & 86 & $2.0 \times 10^{-16}$ & \multirow{2}{*}{0.13} & 0.20 & 54 & $3.2 \times 10^{-16}$ & \multirow[t]{2}{*}{0.05} \\
\hline & 27.7 & 0.36 & 98 & $1.7 \times 10^{-16}$ & & 0.17 & 47 & $3.6 \times 10^{-16}$ & \\
\hline
\end{tabular}

The observed Lyman- $\alpha$ radiation magnitude may be evaluated in terms of densities of the $n=1$ and 2 electronic states of Hydrogen. The intensity observed must be integrated across the diameter of the shock tube:

$$
I=\int \frac{2 h c^{2}}{\lambda^{5}} \frac{g_{1} n_{2}}{g_{2} n_{1}}\left(1-e^{-\phi(\lambda) \frac{h c}{4 \pi \lambda} B_{21} n_{1} D}\right) d \lambda
$$

Assuming collisional broadening to be small in the freestream, this is evaluated with a Doppler lineshape and the result is found to be weakly dependent on translational temperature. In the optically thin limit, the $\mathrm{n}_{2}$ density would need to be on the order of $10^{9} \mathrm{~cm}^{-3}$. However, the radiance is determined to be optically thick for $\mathrm{n}_{1}$ larger than $10^{12}$ $\mathrm{cm}^{-3}$ (or, equivalently, $\mathrm{T}_{\mathrm{e}}<15,000 \mathrm{~K}$ ). This corresponds to approximately $10^{-5}$ times the $\mathrm{H}$ atom density in the shock layer and $10^{-4}$ times the freestream density. Given the diffusion coefficients discussed above, it is reasonable to assume that the density is above this limit. Therefore, the radiance observed is only sensitive to the ratio of $\mathrm{n}_{2} / \mathrm{n}_{1}$, or equivalently the effective temperature, $T_{e}^{(1,2)}$. This effective temperature is shown as a function of pre-shock length in Figure 14. The effective temperature increases from near 10,000-12,000 $\mathrm{K}$ at the shock front to $12,000-14,000 \mathrm{~K}$ in front of the shock before decaying out into the freestream.

The final interpretation of the pre-shock radiation will require further study. While the correlation to the diffusion length is suggestive, it is not conclusive as the single exponential decay does not account for the effect of decreasing temperature into the free stream. This result should be supported by CFD simulations which include a sufficient free stream volume to allow diffusion to occur at its natural length scale. The observed decay rates appear too rapid to be attributed to a radiative excitation of the freestream, however this cannot be fully discounted either without simultaneously modeling the drift-diffusion and state to state excitation processes. The fact that the decay length is a small multiple of the mean free path in the freestream may indicate that continuum modelling will be insufficient to capture this trend, and the affect may be better explained by non-continuum methods such as Direct Simulation Monte Carlo (DSMC).[22] 


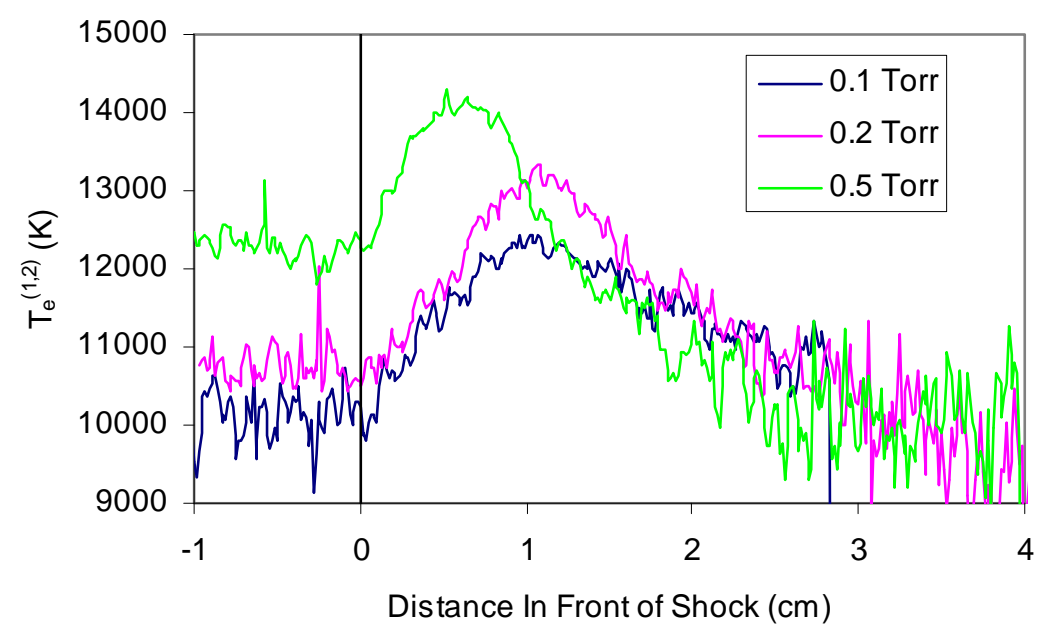

Figure 14. The equivalent temperature between $n=1$ and $n=2$ states of $H$ in front of the shock based on emission intensity. The estimated shock front location has been shifted by no more than $0.5 \mathrm{~cm}$ to place the inflection at position 0.

\section{Conclusions and Recommendations}

These tests have established conditions for future testing of Hydrogen/Helium mixtures in the EAST facility. Previous issues with gas preheating by driver radiation have been eliminated, within detection limits, through the use of thin opaque buffer diaphragms and He driver gas composition. Tests below $25.0 \mathrm{~km} / \mathrm{s}$ display no radiance, as expected, due to the low temperature obtained in hydrogen. The majority of Uranus entries would fall into this category. Between $25-30 \mathrm{~km} / \mathrm{s}$, radiation is observed, but equilibrium levels are not obtained over several centimeters. For most shocks, an induction time is observed, whereby radiance levels rapidly increase from low levels several $\mathrm{cm}$ behind the shock front. This sudden change in radiance is shown to coincide with an increase in electron density and effective electronic temperature. The electron density trend is predicted by existing CFD tools, where the relatively slow ionization rate causes the electron density to gradually build up over the width of the shock. While the agreement is qualitative, simulating the wider range of EAST tests using larger shock stand-offs would provide further verification. The effective temperature trend is not captured by CFD as it is attributed to quasi-steady state phenomena that are typically implemented within the radiation code. Radiance, particularly in the VUV, is observed in front of the nominal shock front. The characteristic distance over which the radiance persists is shown to match the ratio of diffusion to shock velocity and is approximately 2 mean free paths in the freestream. Further study to understand the mechanism is required.

The radiance obtained through NEQAIR with Boltzmann state modeling is shown to overpredict flight-relevant conditions by as much as a factor of 10 . The disagreement is attributed to the absence of a quasi-steady state model. Additional gaps in the NEQAIR modeling capability also identified include the $\mathrm{H}$ atom Stark broadening mechanism and Balmer continuum prediction. It is possible that the QSS approach would improve the continuum underprediction. The shock tube data indicates that, for a $1 \mathrm{~m}$ diameter probe with shock stand-off distances around $2.0-2.5 \mathrm{~cm}$, radiative heating is unlikely to be significant relative to convective heating for most Uranus or Saturn entry trajectories. This is because the ionization rate of hydrogen is slow enough that the electron density does not obtain the critical density necessary over this distance to excite the emitting states. The agency's current tools are expected to overpredict this heating, though the extent to which they will overpredict is not fully determined. Some consideration should also be given to the possibility that trace atmospheric hydrocarbons or ablation products/impurities may "seed" the shock layer with easily ionized species. Percent levels of methane in the atmosphere have been shown to increase radiation significantly [16], which may be relevant for Saturn, but not Uranus, entries. Entries to Neptune or Jupiter, which occur at higher velocity, are expected to have more substantial radiative heating $[16,23,24]$ and would require revisions to the radiative model for accurate predictions. 


\section{References}

1. National Research Council, Vision and Voyages for Planetary Science in the Decade 2013-2022, National Academies Press, 2012.

2. Palmer, G., Prabhu, D., and Cruden, B. A., "Aeroheating Uncertainties in Uranus and Saturn Entries by the Monte Carlo Method," Journal of Spacecraft and Rockets, Vol. 51, No. 3, 2014, pp. 801-814.

3. Dannenberg, R., "A new look at performance capabilities of arc-driven shock tubes," Shock tube and shock wave research; Eleventh International Symposium. Seattle, WA, 1977.

4. Hollis, B., Wright, M., Olejniczak, J., Takashima, N., Sutton, K., and Prabhu, D., "Preliminary Convective-Radiative Heating Environments for a Neptune Aerocapture Mission," AIAA 2004-5177, 16 - 19 August 2004.

5. Bogdanoff, D. W., and Park, C., "Radiative interaction between driver and driven gases in an arc-driven shock tube," Shock Waves, Vol. 12, No. 3, 2002, pp. 205-214.

6. Cruden , B. A., "Absolute Radiation Measurements in Earth and Mars Entry Conditions ", Technical Report RTO-ENAVT-218, NATO.

7. Dannenberg, R. E., "A conical arc driver for high-energy test facilities," AIAA Journal, Vol. 10, 1972, pp. 1692-1694.

8. Conrath, B. J., and Gautier, D., "Saturn Helium Abundance: A Reanalysis of Voyager Measurements," Icarus, Vol. 144, No. 1, 2000, pp. 124-134.

9. Conrath, B., Gautier, D., Hanel, R., Lindal, G., and Marten, A., "The helium abundance of Uranus from Voyager measurements," Journal of Geophysical Research: Space Physics, Vol. 92, No. A13, 1987, pp. 15003-15010.

10. Greenberg, R. B., Cruden, B. A., Grinstead, J. H., and Yeung, D., "Collection optics for imaging spectroscopy of an electric arc shock tube," Proccedings of the SPIE, Novel Optical Systems Design and Optimization XII. Vol. 7429, $2009,74290 \mathrm{H}$.

11. McBride, B. J., and Gordon, S., "Computer program for calculation of complex chemical equilibrium compositions and applications II. User's Manual and Program Description," NASA RP-1311-P2, June 1996.

12. Cruden , B. A., and Brandis, A. M., "Updates to the NEQAIR Radiation Solver," Radiation in High Temperature Gases. St. Andrews, UK, 2014.

13. Cunto, W., Mendoza, C., Ochsenbein, F., and Zeippen, C., "TOPbase at the CDS," The Opacity Project: Selected research papers-Atomic Data Tables for S to Fe, Vol. 2, 1994, p. 53.

14. Gigosos, M. A., and Cardeñoso, V., "New plasma diagnosis tables of hydrogen Stark broadening including ion dynamics," Journal of Physics B-Atomic Molecular and Optical Physics, Vol. 29, No. 20, 1996, pp. 4795-4838.

15. Cruden, B. A., "Electron Density Measurement in Reentry Shocks for Lunar Return," Journal of Thermophysics and Heat Transfer, Vol. 26, No. 2, 2012, pp. 222-230.

16. Park, C., "Nonequilibrium Chemistry and Radiation for Neptune Entry," Journal of Spacecraft and Rockets, Vol. 48, No. 6, 2011, pp. 897-903.

17. Bishop, J., Atreya, S. K., Herbert, F., and Romani, P., "Reanalysis of Voyager 2 UVS Occultations at Uranus: Hydrocarbon Mixing Ratios in the Equatorial Stratosphere," Icarus, Vol. 88, 1990, pp. 448-464.

18. Moses, J., "Photochemistry of Saturn's Atmosphere I. Hydrocarbon Chemistry and Comparisons with ISO Observations," Icarus, Vol. 143, No. 2, 2000, pp. 244-298.

19. Atreya, S. K., "Hydrocarbons and Eddy Mixing in Neptune's Atmosphere," Adv. Spece. Res., Vol. 12, No. 11, 1992, pp. 11-18.

20. Park, C., "Nonequilibrium Ionization and Radiation in Hydrogen-Helium Mixtures," Journal of Thermophysics and Heat Transfer, Vol. 26, No. 2, 2012, pp. 231-243.

21. Cruden, B. A., Brandis, A. M., and Prabhu, D. K., "Compositional Dependence of Radiance in $\mathrm{CO}_{2} / \mathrm{N}_{2} / \mathrm{Ar} \mathrm{Systems,"}$ AIAA 2013-2502.

22. George, J., and Boyd, I., "Numerical investigation of hydrogen plume flows using unstructured grids," 1998.

23. Matsuyama, S., Ohnishi, N., Sasoh, A., and Sawada, K., "Numerical simulation of Galileo probe entry flowfield with radiation and ablation," Journal of Thermophysics and Heat Transfer, Vol. 19, No. 1, 2005, pp. 28-35.

24. Park, C., "Stagnation-region heating environment of the Galileo probe," Journal of Thermophysics and Heat Transfer, Vol. 23, No. 3, 2009, pp. 417-424. 